\title{
GLOBAL SMALL SOLUTION TO THE 2D MHD SYSTEM WITH A VELOCITY DAMPING TERM
}

\author{
JIAHONG WU ${ }^{1}$, YIFEI WU ${ }^{2}$, AND XIAOJING XU²
}

\begin{abstract}
This paper studies the global well-posedness of the incompressible magnetohydrodynamic (MHD) system with a velocity damping term. We establish the global existence and uniqueness of smooth solutions when the initial data is close to an equilibrium state. In addition, explicit large-time decay rates for various Sobolev norms of the solutions are also given.
\end{abstract}

\section{INTRODUCTION}

This paper examines the global (in time) existence and uniqueness of solutions to the 2D magnetohydrodynamic (MHD) system with a velocity damping term, namely

$$
\left\{\begin{array}{l}
\partial_{t} \vec{u}+\vec{u} \cdot \nabla \vec{u}+\vec{u}+\nabla P=-\nabla \cdot(\nabla \phi \otimes \nabla \phi), \quad(t, x, y) \in \mathbb{R}_{+} \times \mathbb{R} \times \mathbb{R}, \\
\partial_{t} \phi+\vec{u} \cdot \nabla \phi=0, \\
\nabla \cdot \vec{u}=0, \\
\left.\vec{u}\right|_{t=1}=\vec{u}_{0}(x, y),\left.\quad \phi\right|_{t=1}=\phi_{0}(x, y),
\end{array}\right.
$$

where $\vec{u}=(u, v)$ represents the $2 \mathrm{D}$ velocity field, $P$ the pressure and $\phi$ the magnetic stream function, and $\nabla \phi \otimes \nabla \phi$ denotes the tensor product. (1.1) is formally equivalent to the 2D MHD equations given by

$$
\left\{\begin{array}{l}
\partial_{t} \vec{u}+\vec{u} \cdot \nabla \vec{u}+\vec{u}+\nabla P=-\frac{1}{2} \nabla\left(|\vec{b}|^{2}\right)+\vec{b} \cdot \nabla \vec{b} \\
\partial_{t} \vec{b}+\vec{u} \cdot \nabla \vec{b}=\vec{b} \cdot \nabla \vec{u} \\
\nabla \cdot \vec{b}=\nabla \cdot \vec{u}=0 .
\end{array}\right.
$$

In fact, $\nabla \cdot \vec{b}=0$ implies that $\vec{b}=\nabla^{\perp} \phi \equiv\left(\partial_{y} \phi,-\partial_{x} \phi\right)$ for a scalar function $\phi$ and, with this substitution, (1.2) is reduced to (1.1). The MHD equations, modeling electrically conducting fluid in the presence of a magnetic field, consist essentially of the interaction between the fluid velocity and the magnetic field. Electric currents induced in the fluid as a result of its motion modify the field; at the same time their flow in the magnetic field leads to mechanical forces which modify the motion. The MHD equations underly many phenomena such as the geomagnetic dynamo in geophysics and solar winds and solar flares in astrophysics (see, e.g., [1, 9, 22]).

Mathematically the MHD equations can be extremely difficult to analyze due to the nonlinear coupling between the forced Navier-Stokes equations and the induction equation. In fact, it remains an outstanding open problem whether solutions to the 2D

Date: April 8, 2018. 
MHD equations

$$
\left\{\begin{array}{l}
\partial_{t} \vec{u}+\vec{u} \cdot \nabla \vec{u}+\nabla P=-\frac{1}{2} \nabla\left(|\vec{b}|^{2}\right)+\vec{b} \cdot \nabla \vec{b} \\
\partial_{t} \vec{b}+\vec{u} \cdot \nabla \vec{b}=\vec{b} \cdot \nabla \vec{u} \\
\nabla \cdot \vec{b}=\nabla \cdot \vec{u}=0
\end{array}\right.
$$

exist for all time or they blow up in a finite time. One main difficulty is the lack of global (in time) bounds for the Sobolev norms of the solutions. Adding a velocity damping term does not appear to be sufficient to overcome this difficulty and our aim here is at small global smooth solutions. Since the equation of $\phi$ in (1.1) is a transport equation without any damping or dissipation, it is a very involved problem to establish the global well-posedness of (1.1) even under the assumption that the initial data is small.

The global regularity problem on the 2D MHD equations with partial dissipation or partial damping has attracted considerable interests in the last few years and progress has been made for some cases. The anisotropic 2D MHD equations with horizontal dissipation and vertical magnetic diffusion were recently examined by Cao and $\mathrm{Wu}$ and shown to possess global classical solutions for any sufficiently smooth data [5]. Advances have also been achieved for the case when the dissipation and the magnetic diffusion are both in the horizontal direction $([3,4])$. F. Lin, L. Xu and P. Zhang recently studied the MHD equations with the Laplacian dissipation in the velocity equation but without magnetic diffusion and, remarkably, they were able to establish the global existence of small solutions after translating the magnetic field by a constant vector ([20, 21, 29]). Their approach reformulates the system in Lagrangian coordinates and estimates the Lagrangian velocity through the anisotropic Littlewood-Paley theory and anisotropic Besov space techniques. The partial dissipation case when only the magnetic diffusion is present has also been examined and the global $H^{1}$ weak solutions have been established (see, e.g., [5, 18]). In addition, if we increase the magnetic diffusion from the Laplacian operator to the fractional Laplacian operator $(-\Delta)^{\beta}$ with $\beta>1$, then the resulting MHD equations do have global regular solutions $([6$, 16]). Many more recent results on the MHD equations with partial or fractional dissipation can be bound in the references [7, 8, 12, 13, 15, 17, 24, 25, 26, 27, 28, 30, 31, 32, 33.

The contribution of this paper is the global existence and uniqueness of solutions of (1.1) with sufficiently smooth initial data $\left(u_{0}, \phi_{0}\right)$ close to the equilibrium state $(0, y)$. This work is partially inspired by [20]. Our approach here exploits the time decay properties of the solution kernels to a linear differential equation which, with suitable nonlinear forcing terms, governs the translated version of (1.1). We now give a more precise account of our ideas. Setting

$$
\phi=\psi+y
$$

converts (1.1) into the following equivalent system of equations for $(u, v, \psi)$,

$$
\left\{\begin{array}{l}
\partial_{t} u+u \partial_{x} u+v \partial_{y} u+u+\partial_{x} \tilde{P}=-\Delta \psi \partial_{x} \psi, \\
\partial_{t} v+u \partial_{x} v+v \partial_{y} v+v+\partial_{y} \tilde{P}=-\Delta \psi-\Delta \psi \partial_{y} \psi, \\
\partial_{t} \psi+u \partial_{x} \psi+v \partial_{y} \psi+v=0, \\
\partial_{x} u+\partial_{y} v=0
\end{array}\right.
$$


where $\tilde{P}=P+\frac{1}{2}|\nabla \phi|^{2}$. Applying $\nabla \cdot \vec{u}=0$ to eliminate the pressure term yields

$$
\begin{aligned}
& \partial_{t} u+u-\partial_{x y} \psi=\Pi_{1}, \\
& \partial_{t} v+v+\partial_{x x} \psi=\Pi_{2}, \\
& \partial_{t} \psi+u \partial_{x} \psi+v \partial_{y} \psi+v=0,
\end{aligned}
$$

where

$$
\begin{aligned}
& \Pi_{1}=-\vec{u} \cdot \nabla u+\partial_{x} \Delta^{-1} \nabla \cdot(\vec{u} \cdot \nabla \vec{u})-\Delta \psi \partial_{x} \psi+\partial_{x} \Delta^{-1} \nabla \cdot(\Delta \psi \nabla \psi), \\
& \Pi_{2}=-\vec{u} \cdot \nabla v+\partial_{y} \Delta^{-1} \nabla \cdot(\vec{u} \cdot \nabla \vec{u})-\Delta \psi \partial_{y} \psi+\partial_{y} \Delta^{-1} \nabla \cdot(\Delta \psi \nabla \psi) .
\end{aligned}
$$

Taking the time derivative on the equations (1.5)-(1.7), we obtain

$$
\left\{\begin{array}{l}
\partial_{t t} u+\partial_{t} u-\partial_{x x} u=F_{1}, \\
\partial_{t t} v+\partial_{t} v-\partial_{x x} v=F_{2}, \\
\partial_{t t} \psi+\partial_{t} \psi-\partial_{x x} \psi=F_{0}, \\
\left.\vec{u}\right|_{t=1}=\vec{u}_{0}(x, y),\left.\quad \vec{u}_{t}\right|_{t=1}=\vec{u}_{1}(x, y), \\
\left.\psi\right|_{t=1}=\psi_{0}(x, y),\left.\quad \psi_{t}\right|_{t=1}=\psi_{1}(x, y),
\end{array}\right.
$$

where $\vec{u}_{1}=\left(u_{1}(x, y), v_{1}(x, y)\right), \psi_{0}=\phi_{0}-y$, and

$$
\begin{aligned}
& u_{1}=\left.\left(-u+\partial_{x y} \psi+\Pi_{1}\right)\right|_{t=1}, \\
& v_{1}=\left.\left(-v-\partial_{x x} \psi+\Pi_{2}\right)\right|_{t=1}, \\
& \psi_{1}=\left.\left(-u \partial_{x} \psi-v \partial_{y} \psi-v\right)\right|_{t=1},
\end{aligned}
$$

and

$$
\begin{aligned}
& F_{0}=-\vec{u} \cdot \nabla \psi-\partial_{t}(\vec{u} \cdot \nabla \psi)-\Pi_{2}, \\
& F_{1}=\partial_{t} \Pi_{1}-\partial_{x y}(\vec{u} \cdot \nabla \psi), \\
& F_{2}=\partial_{t} \Pi_{2}+\partial_{x x}(\vec{u} \cdot \nabla \psi) .
\end{aligned}
$$

The structure of the linear part in (1.10) plays a crucial role in ensuring the global existence of small solutions. In fact, the solution kernels of the linear equation decay in time in suitable spatial functional settings. Let us be more accurate. As detailed in Section 2, the solution of the linear equation

$$
\partial_{t t} \Phi+\partial_{t} \Phi-\partial_{x x} \Phi=0
$$

with the initial data

$$
\Phi(0, x, y)=\Phi_{0}(x, y), \quad \Phi_{t}(0, x, y)=\Phi_{1}(x, y)
$$

can be written as

$$
\Phi(t, x, y)=K_{0}\left(t, \partial_{x}\right) \Phi_{0}+K_{1}\left(t, \partial_{x}\right)\left(\frac{1}{2} \Phi_{0}+\Phi_{1}\right)
$$

where the solution operators $K_{1}$ and $K_{2}$ are explicitly derived in Section 2 , By Duhamel's Principle, the solution of the inhomogeneous equation

$$
\partial_{t t} \Phi+\partial_{t} \Phi-\partial_{x x} \Phi=F
$$

with initial data $\Phi(1, x)=\Phi_{0}, \partial_{t} \Phi(1, x)=\Phi_{1}$ is given by

$$
\Phi(t, x, y)=K_{0}\left(t, \partial_{x}\right) \Phi_{0}+K_{1}\left(t, \partial_{x}\right)\left(\frac{1}{2} \Phi_{0}+\Phi_{1}\right)
$$




$$
+\int_{1}^{t} K_{1}\left(t-s, \partial_{x}\right) F(s, x, y) d s .
$$

By letting $\Phi=(u, v, \psi)$ and $F=\left(F_{0}, F_{1}, F_{2}\right)$, (1.14) gives an integral representation of (1.10). Thanks to the time decay properties of $K_{1}$ and $K_{2}$ (established in Section 2), the nonlinear parts in (1.10) remain small and the solution map is a contraction for all time. More details will be unfolded in the subsequent sections.

To state our main result, we introduce the functional settings. Let $X_{0}$ be the Banach space defined by the norm

$$
\left\|\left(\vec{u}_{0}, \psi_{0}\right)\right\|_{X_{0}}=\left\|\langle\nabla\rangle^{N}\left(\vec{u}_{0}, \nabla \psi_{0}\right)\right\|_{L_{x y}^{2}}+\left\|\langle\nabla\rangle^{6+}\left(\vec{u}_{0}, \psi_{0}\right)\right\|_{L_{x y}^{1}}+\left\|\langle\nabla\rangle^{6+}\left(\vec{u}_{1}, \psi_{1}\right)\right\|_{L_{x y}^{1}} .
$$

where $\langle\nabla\rangle=(I-\Delta)^{\frac{1}{2}}$ and $a+$ denotes $a+\epsilon$ for any small $\epsilon$. For notational convenience, we also write

$$
\|f\|_{q}=\|f\|_{L_{x y}^{q}}, \quad 1 \leq q \leq \infty .
$$

Now we define our working space as $X$ with its norm given by

$$
\begin{aligned}
\|(\vec{u}, \psi)\|_{X}=\sup _{t \geq 1} & \left\{t^{-\varepsilon}\left\|\langle\nabla\rangle^{N}(\vec{u}(t), \nabla \psi(t))\right\|_{2}+t^{\frac{1}{4}}\left\|\langle\nabla\rangle^{3} \psi\right\|_{2}\right. \\
& +t^{\frac{3}{2}}\left\|\langle\nabla\rangle \partial_{x x} \psi\right\|_{\infty}+t^{\frac{5}{4}}\left\|\langle\nabla\rangle^{3} \partial_{x x} \psi\right\|_{2}+t^{\frac{3}{2}}\left\|\partial_{x x x} \psi\right\|_{2} \\
& \left.+t^{\frac{3}{2}}\left\|\partial_{t} \vec{u}\right\|_{\infty}+t^{\frac{5}{4}}\left\|\langle\nabla\rangle \partial_{t} \vec{u}\right\|_{2}+t\left\|\langle\nabla\rangle \partial_{x} \vec{u}\right\|_{\infty}+t^{\frac{3}{2}}\left\|\partial_{x} \partial_{t} v\right\|_{2}\right\} .
\end{aligned}
$$

Here $N$ is a big positive integer and $\varepsilon>0$ is a small parameter. For the sake of the clarity of our presentation, we intentionally avoid the tedious calculations needed for providing an accurate range of $N$. However, sufficiently large $N$ and small $\varepsilon>0$, say $N=20$ and $\varepsilon=0.01$, would serve our purpose.

Our main result can then be stated as follows. We use $A \lesssim B$ or $B \gtrsim A$ to denote the statement that $A \leq C B$ for some absolute constant $C>0$.

Theorem 1.1. Let $\psi=\phi-y$ and $\psi_{0}=\phi_{0}-y$. Then there exists a small constant $\varepsilon_{0}>0$ such that, if the initial data $\left(\vec{u}_{0}, \phi_{0}\right)$ satisfying $\left\|\left(\vec{u}_{0}, \psi_{0}\right)\right\|_{X_{0}} \leq \varepsilon_{0}$, then there exists a unique global solution $(u, v, \phi, P)$ to the system (1.1) with

$$
(u, v, \phi) \in X, \quad P \in C\left([1, \infty) ; H^{N}\left(\mathbb{R}^{2}\right)\right) .
$$

Moreover, the following decay estimates hold

$$
\|u(t)\|_{L_{x y}^{\infty}} \lesssim \varepsilon_{0} t^{-1} ; \quad\|v(t)\|_{L_{x y}^{\infty}} \lesssim \varepsilon_{0} t^{-\frac{3}{2}} ; \quad\|\psi(t)\|_{L_{x y}^{\infty}} \lesssim \varepsilon_{0} t^{-\frac{1}{2}} ; \quad\|P(t)\|_{L_{x y}^{\infty}} \lesssim \varepsilon_{0} t^{-\frac{1}{2}} .
$$

The proof of Theorem 1.1 relies on the following lemma, which can be deduced from a standard continuity argument (see, e.g., Theorem 4 in [2]).

Lemma 1.2. Assume the initial data $\left(\vec{u}_{0}, \psi_{0}\right) \in X_{0}$. Suppose that $(\vec{u}, \psi)$ given by (1.14) namely the integral representation of (1.10), satisfies

$$
\left.\|(\vec{u}, \psi)\|_{X} \lesssim\left\|\left(\vec{u}_{0}, \psi_{0}\right)\right\|_{X_{0}}+Q(\| \vec{u}, \psi) \|_{X}\right),
$$

where $Q(a) \geq C a^{\beta}$ for $a \lesssim 1$ and $\beta>1$. Then there exists $r_{0}>0$ such that, if

$$
\left\|\left(\vec{u}_{0}, \psi_{0}\right)\right\|_{X_{0}} \lesssim r_{0}
$$


then (1.10) has a unique global solution $(\vec{u}, \psi) \in X$ and

$$
\|(\vec{u}, \psi)\|_{X} \lesssim 2 r_{0}
$$

In addition, to facilitate the proof, we introduce an auxiliary functional space, in which more terms with explicit time decay estimates are included. Let

$$
\begin{aligned}
\|(\vec{u}, \psi)\|_{Y}= & \|(\vec{u}, \psi)\|_{X}+\sup _{t \geq 1}\left\{t^{\frac{1}{2}}\left\|\langle\nabla\rangle^{2} \psi\right\|_{\infty}+t^{\frac{3}{4}}\left\|\langle\nabla\rangle \partial_{x} \psi\right\|_{2}+t\left\|\partial_{x}\langle\nabla\rangle^{3} \psi\right\|_{\infty}\right. \\
& \left.+t\|u\|_{\infty}+t^{\frac{3}{2}}\|v\|_{\infty}+t^{\frac{3}{4}}\|u\|_{2}+t^{\frac{5}{4}}\left\|\partial_{x} u\right\|_{2}+t^{\frac{5}{4}}\|v\|_{2}\right\} .
\end{aligned}
$$

Roughly speaking, the decay rates of the extra terms in the $Y$-norm obey the following rules:

$$
L^{\infty} \sim t^{-\frac{1}{2}} ; \quad L^{2} \sim t^{-\frac{1}{4}} ; \quad \partial_{x} \sim t^{-\frac{1}{2}} ; \quad \partial_{t} \sim \partial_{x x} ; \quad u \sim \partial_{x} \sim t^{-\frac{1}{2}} ; \quad v \sim \partial_{x x} \sim t^{-1} .
$$

As we show in Section 3 , the norms $\|(\vec{u}, \psi)\|_{Y}$ and $\|(\vec{u}, \psi)\|_{X}$ are related through the following lemma.

Lemma 1.3. Let the spaces $X, Y$ and their norms be defined as above. Then

$$
\|(\vec{u}, \psi)\|_{Y} \lesssim\|(\vec{u}, \psi)\|_{X}+Q\left(\|(\vec{u}, \psi)\|_{X}\right) .
$$

As a consequence of Lemma 1.3. to prove (1.16), it is enough to verify

$$
\|(\vec{u}, \psi)\|_{X} \lesssim\left\|\left(\vec{u}_{0}, \psi_{0}\right)\right\|_{X_{0}}+Q\left(\|(\vec{u}, \psi)\|_{Y}\right) .
$$

Therefore, the proof of Theorem 1.1 is then reduced to establishing (1.17) and our main effort is devoted to achieving this goal. This is a long process and involves several major components. The first consists of crucial and sharp decay estimates and four tool lemmas on the kernels. The second is the bounds on the nonlinearities $F_{0}, F_{1}$ and $F_{2}$ by treating differently the lower and higher frequencies of their terms. The third involves the estimates of each member in the $X$-norm in (1.15) through the first two components.

The rest of the paper is divided into six sections and an appendix. The second section derives the solution kernel of the linear equation and represents the solution of (1.10) in an integral form through the Duhamel formula. Crucial decay estimates for the solution kernels and four tool lemmas to be used repeatedly are also presented in this section. Section 3 proves Lemma 1.3. The rest of the sections are devoted to proving (1.17). Section 4 bounds $t^{-\varepsilon}\left\|\langle\nabla\rangle^{N}(\vec{u}(t), \nabla \psi(t))\right\|_{2}$ through energy estimates, which give a control of the first term in the definition of the norm of $X$. Section 5 provides suitable estimates for the nonlinear terms $F_{0}, F_{1}$ and $F_{2}$. To obtain these estimates, we decompose the terms involved to high and low frequencies and apply the results from the second section and an inequality involving the Riesz transform (Lemma 5.1). With the estimates for $F_{0}, F_{1}$ and $F_{2}$ at our disposal, Sections 6 and 7 continue the proof of (1.17) by repeatedly applying the tool lemmas in the second section and the estimates for $F_{0}, F_{1}$ and $F_{2}$. The appendix serves four purposes. It gives an explicit representation of $\Pi_{1}$ and $\Pi_{2}$. The key point of this representation is that each term is written in a way that it possesses as many directives in the $x$-direction as possible. As seen from Section 2, the more $x$-derivatives a term has, the faster it decays in time. This point has played an important role in the estimates of $F_{0}, F_{1}$ and $F_{2}$ in Section 5 . It also provides the proofs of Lemma 5.1 and Lemma 5.5. Finally, the properties of the pressure are also given here. 


\section{Preliminary}

This section is divided into two subsections. The first subsection derives the integral formulation (1.14) with explicit representations for $K_{1}$ and $K_{2}$. In addition, key decay estimates for $K_{1}$ and $K_{2}$ are also obtained here. The second subsection proves several tool lemmas to be used in the proof of Theorem 1.1 .

2.1. Linear operators. We consider the linear equation

$$
\partial_{t t} \Phi+\partial_{t} \Phi-\partial_{x x} \Phi=0
$$

with the initial data

$$
\Phi(0, x, y)=\Phi_{0}(x, y), \Phi_{t}(0, x, y)=\Phi_{1}(x, y) .
$$

Taking the Fourier transform of (2.1) yields

$$
\partial_{t t} \hat{\Phi}+\partial_{t} \hat{\Phi}+\xi^{2} \hat{\Phi}=0
$$

where the Fourier transform $\hat{\Phi}$ is defined as

$$
\hat{\Phi}(t, \xi, \eta)=\int_{\mathbb{R}^{2}} e^{-i x \xi-i y \eta} \Phi(t, x, y) d x d y
$$

Solving (2.2) by a simple ODE theory, we have

$$
\begin{aligned}
\hat{\Phi}(t, \xi, \eta)= & \frac{1}{2}\left(e^{\left(-\frac{1}{2}+\sqrt{\frac{1}{4}-\xi^{2}}\right) t}+e^{\left(-\frac{1}{2}-\sqrt{\frac{1}{4}-\xi^{2}}\right) t}\right) \widehat{\Phi_{0}}(\xi, \eta) \\
& +\frac{1}{2 \sqrt{\frac{1}{4}-\xi^{2}}}\left(e^{\left(-\frac{1}{2}+\sqrt{\frac{1}{4}-\xi^{2}}\right) t}-e^{\left(-\frac{1}{2}-\sqrt{\frac{1}{4}-\xi^{2}}\right) t}\right)\left(\frac{1}{2} \widehat{\Phi_{0}}(\xi, \eta)+\widehat{\Phi_{1}}(\xi, \eta)\right) .
\end{aligned}
$$

Definition 2.1. Let the operators $K_{0}\left(t, \partial_{x}\right)$ and $K_{1}\left(t, \partial_{x}\right)$ be defined as

$$
\left.\widehat{K_{0}} \widehat{\left(t, \partial_{x}\right.}\right) f(t, \xi, \eta)=\frac{1}{2}\left(e^{\left(-\frac{1}{2}+\sqrt{\frac{1}{4}-\xi^{2}}\right) t}+e^{\left(-\frac{1}{2}-\sqrt{\frac{1}{4}-\xi^{2}}\right) t}\right) \hat{f}(t, \xi, \eta) ;
$$

and

$$
\left.\widehat{K_{1}\left(t, \partial_{x}\right.}\right) f(t, \xi, \eta)=\frac{1}{2 \sqrt{\frac{1}{4}-\xi^{2}}}\left(e^{\left(-\frac{1}{2}+\sqrt{\frac{1}{4}-\xi^{2}}\right) t}-e^{\left(-\frac{1}{2}-\sqrt{\frac{1}{4}-\xi^{2}}\right) t}\right) \hat{f}(t, \xi, \eta),
$$

where $\sqrt{-1}=i$.

By Definition 2.1, the solution $\Phi$ of the equation (2.1) is written as

$$
\Phi(t, x, y)=K_{0}\left(t, \partial_{x}\right) \Phi_{0}+K_{1}\left(t, \partial_{x}\right)\left(\frac{1}{2} \Phi_{0}+\Phi_{1}\right) .
$$

Moreover, consider the inhomogeneous equation,

$$
\partial_{t t} \Phi+\partial_{t} \Phi-\partial_{x x} \Phi=F
$$

with initial data $\Phi(1, x)=\Phi_{0}, \partial_{t} \Phi(1, x)=\Phi_{1}$. Then we have the following standard Duhamel formula,

$$
\Phi(t, x, y)=K_{0}\left(t, \partial_{x}\right) \Phi_{0}+K_{1}\left(t, \partial_{x}\right)\left(\frac{1}{2} \Phi_{0}+\Phi_{1}\right)+\int_{1}^{t} K_{1}\left(t-s, \partial_{x}\right) F(s, x, y) d s .
$$

In the following, we present some decay estimates on $K_{0}, K_{1}$. 
Lemma 2.2. Let $K_{0}, K_{1}$ be defined in Definition [2.1. Then for any $\alpha \geq 0,0 \leq \beta \leq 2$, $1 \leq q \leq \infty, i=0,1$,

1) $\left\||\xi|^{\alpha} \widehat{K_{i}}(t, \cdot)\right\|_{L_{\xi}^{q}\left(|\xi| \leq \frac{1}{2}\right)} \lesssim\langle t\rangle^{-\frac{1}{2}\left(\frac{1}{q}+\alpha\right)}$

2) $\left\||\xi|^{-\beta} \partial_{t} \widehat{K_{i}}(t, \cdot)\right\|_{L_{\xi}^{q}\left(|\xi| \leq \frac{1}{2}\right)} \lesssim\langle t\rangle^{-1-\frac{1}{2}\left(\frac{1}{q}-\beta\right)}$

3) $\left|\widehat{K}_{i}(t, \xi)\right| \lesssim e^{-\frac{1}{2} t}$, for any $|\xi| \geq \frac{1}{2}, i=0,1$;

4) $\left|\langle\xi\rangle^{-1} \partial_{t} \widehat{K_{0}}(t, \xi)\right|,\left|\partial_{t} \widehat{K_{1}}(t, \xi)\right| \lesssim e^{-\frac{1}{2} t}$, for any $|\xi| \geq \frac{1}{2}$.

Proof. Since the decay properties of the operators $K_{0}, K_{1}$ are distinct between low and high frequencies, we will split its frequency into the following two parts:

$$
|\xi| \leq \frac{1}{2} ; \quad|\xi|>\frac{1}{2}
$$

In the following, we will analyze the two parts separately.

Case I: $|\xi| \leq \frac{1}{2}$.

According to the expressions of $K_{0}, K_{1}$ as follows

$$
\widehat{K_{0}}(t, \xi)=\frac{1}{2}\left(e^{\left(-\frac{1}{2}+\sqrt{\frac{1}{4}-\xi^{2}}\right) t}+e^{\left(-\frac{1}{2}-\sqrt{\frac{1}{4}-\xi^{2}}\right) t}\right)
$$

and

$$
\widehat{K_{1}}(t, \xi)=\frac{1}{2 \sqrt{\frac{1}{4}-\xi^{2}}}\left(e^{\left(-\frac{1}{2}+\sqrt{\frac{1}{4}-\xi^{2}}\right) t}-e^{\left(-\frac{1}{2}-\sqrt{\frac{1}{4}-\xi^{2}}\right) t}\right),
$$

we obtain, for $|\xi| \leq \frac{1}{2}$,

$$
-\frac{1}{2}+\sqrt{\frac{1}{4}-\xi^{2}}=\frac{-\xi^{2}}{\frac{1}{2}+\sqrt{\frac{1}{4}-\xi^{2}}} \leq-\xi^{2} ; \quad-\frac{1}{2}-\sqrt{\frac{1}{4}-\xi^{2}} \leq-\xi^{2} .
$$

Then we get

$$
0 \leq \widehat{K_{0}}(t, \xi) \leq e^{-t \xi^{2}}
$$

Therefore,

$$
\begin{aligned}
|\xi|^{\alpha} \widehat{K_{0}}(t, \xi) & \leq|\xi|^{\alpha} e^{-t \xi^{2}} \lesssim\langle t\rangle^{-\frac{\alpha}{2}} \\
\left\||\xi|^{\alpha} \widehat{K_{0}}(t, \xi)\right\|_{L_{\xi}^{1}\left(|\xi| \leq \frac{1}{2}\right)} & \leq \int_{|\xi| \leq \frac{1}{2}}|\xi|^{\alpha} \widehat{K_{0}}(t, \xi) d \xi \leq \int_{|\xi| \leq \frac{1}{2}}|\xi|^{\alpha} e^{-t \xi^{2}} d \xi \lesssim\langle t\rangle^{-\frac{1}{2}(1+\alpha)}
\end{aligned}
$$

So the estimate 1) of Lemma 2.2 for $K_{0}$ follows from interpolation. Moreover,

$$
\begin{aligned}
& \dot{K}_{0}(t)=-\frac{1}{2} K_{0}(t)+\left(\frac{1}{4}+\partial_{x x}\right) K_{1}(t) ; \\
& \dot{K}_{1}(t)=K_{0}(t)-\frac{1}{2} K_{1}(t) .
\end{aligned}
$$

Thus,

$$
\begin{aligned}
\partial_{t} \widehat{K_{0}}(t, \xi) & =-\frac{1}{2} \widehat{K_{0}}(t, \xi)+\left(\frac{1}{4}-\xi^{2}\right) \widehat{K_{1}}(t, \xi) \\
& =\frac{1}{4}\left(\sqrt{1-4 \xi^{2}}-1\right) e^{\left(-\frac{1}{2}+\sqrt{\frac{1}{4}-\xi^{2}}\right) t}-\frac{1}{4}\left(\sqrt{1-4 \xi^{2}}+1\right) e^{\left(-\frac{1}{2}-\sqrt{\frac{1}{4}-\xi^{2}}\right) t}
\end{aligned}
$$


So we have for any $0 \leq \beta \leq 2$,

$$
\left.\left.|| \xi\right|^{-\beta} \partial_{t} \widehat{K_{0}}(t, \xi)|\lesssim| \xi\right|^{2-\beta} e^{-t \xi^{2}}
$$

Rough speaking, $\partial_{t} \widehat{K_{0}}(t, \xi) \sim \xi^{2} \widehat{K_{0}}(t, \xi)$, and thus we have

$$
\left\||\xi|^{-\beta} \partial_{t} \widehat{K_{0}}(t, \cdot)\right\|_{L_{\xi}^{q}\left(|\xi| \leq \frac{1}{2}\right)} \lesssim\langle t\rangle^{-1-\frac{1}{2}\left(\frac{1}{q}-\beta\right)} .
$$

Similarly, we can obtain

$$
0 \leq \widehat{K_{1}}(t, \xi) \leq e^{-t \xi^{2}} ; \quad\left|\partial_{t} \widehat{K_{1}}(t, \xi)\right| \lesssim \xi^{2} e^{-t \xi^{2}}
$$

Thus for $K_{1}$, we have the same estimates as $K_{0}$. Hence, we deduce the estimates 1) and 2) of Lemma 2.2.

Case II: $|\xi|>\frac{1}{2}$.

For this case, the expressions of $K_{0}, K_{1}$ can be written as

$$
\widehat{K_{0}}(t, \xi)=\frac{1}{2}\left(e^{\left(-\frac{1}{2}+i \sqrt{\xi^{2}-\frac{1}{4}}\right) t}+e^{\left(-\frac{1}{2}-i \sqrt{\xi^{2}-\frac{1}{4}}\right) t}\right)
$$

and

$$
\widehat{K_{1}}(t, \xi)=\frac{1}{2 i \sqrt{\xi^{2}-\frac{1}{4}}}\left(e^{\left(-\frac{1}{2}+i \sqrt{\xi^{2}-\frac{1}{4}}\right) t}-e^{\left(-\frac{1}{2}-i \sqrt{\xi^{2}-\frac{1}{4}}\right) t}\right) .
$$

By virtue of the expression of $K_{0}, K_{1}$, then we get, for any $|\xi|>\frac{1}{2}$,

$$
\left|\widehat{K_{0}}(t, \xi)\right|,\left|\widehat{K_{1}}(t, \xi)\right| \lesssim e^{-\frac{1}{2} t}
$$

Further, by (2.6) and (2.7), we also have

$$
\left|\langle\xi\rangle^{-1} \partial_{t} \widehat{K_{0}}(t, \xi)\right|,\left|\partial_{t} \widehat{K_{1}}(t, \xi)\right| \lesssim e^{-\frac{1}{2} t}
$$

Hence we complete the proof of Lemma 2.2.

2.2. Tool lemmas. To prove (1.17), we need several lemmas. In the following, $\mathcal{S}$ denotes the Schwartz class and $\|f\|_{L_{x}^{p} L_{y}^{q}} \equiv\|\| f\left\|_{L_{y}^{q}}\right\|_{L_{x}^{p}}$.

Lemma 2.3. Let $K\left(t, \partial_{x}\right)$ denote a Fourier multiplier operator with

$$
\|\widehat{K}(t, \xi)\|_{L_{\xi}^{1}}<\infty .
$$

Then, for any space-time Schwartz function $f$,

$$
\left\|K\left(t, \partial_{x}\right) f\right\|_{L_{x y}^{\infty}} \lesssim\|\widehat{K}(t, \xi)\|_{L_{\xi}^{1}}\left\|\partial_{y} f\right\|_{L_{x y}^{1}} .
$$

Proof. For any $g \in \mathcal{S}(\mathbb{R})$, we have

$$
\|g\|_{L^{\infty}(\mathbb{R})} \lesssim\left\|g^{\prime}\right\|_{L^{1}(\mathbb{R})} .
$$

Then, by the inequality above and Young's inequality, we have

$$
\begin{aligned}
\left\|K\left(t, \partial_{x}\right) f\right\|_{L_{x y}^{\infty}} & \lesssim\left\|K\left(t, \partial_{x}\right) \partial_{y} f\right\|_{L_{x}^{\infty} L_{y}^{1}} \\
& \lesssim\left\|K\left(t, \partial_{x}\right) \partial_{y} f\right\|_{L_{y}^{1} L_{x}^{\infty}} \\
& \lesssim\left\|\widehat{K}(t, \xi) \mathcal{F}_{\xi}\left(\partial_{y} f\right)(t, \xi, y)\right\|_{L_{\xi}^{1}} \|_{L_{y}^{1}}
\end{aligned}
$$




$$
\begin{aligned}
& \lesssim\|\widehat{K}(t, \xi)\|_{L_{\xi}^{1}}\|\| \mathcal{F}_{\xi}\left(\partial_{y} f\right)(t, \xi, y)\left\|_{L_{\xi}^{\infty}}\right\|_{L_{y}^{1}} \\
& \lesssim\|\widehat{K}(t, \xi)\|_{L_{\xi}^{1}}\|\| \partial_{y} f(t, x, y)\left\|_{L_{x}^{1}}\right\|_{L_{y}^{1}} \\
& =\|\widehat{K}(t, \xi)\|_{L_{\xi}^{1}}\left\|\partial_{y} f\right\|_{L_{x y}^{1}} .
\end{aligned}
$$

This proves Lemma 2.3 ,

Lemma 2.4. Assume that $\|\widehat{K}(t, \cdot)\|_{L^{\infty}}$ is bounded. Then, for any space-time Schwartz function $f$, and any $\epsilon>0$,

$$
\left\|K\left(t, \partial_{x}\right) f\right\|_{L_{x y}^{\infty}} \lesssim\|\widehat{K}(t, \xi)\|_{L_{\xi}^{\infty}}\left\|\partial_{y}\langle\nabla\rangle^{1+\epsilon} f\right\|_{L_{x y}^{1}}
$$

Proof. By (2.9), Sobolev's inequality and Plancherel's identity, we have

$$
\begin{aligned}
\left\|K\left(t, \partial_{x}\right) f\right\|_{L_{x y}^{\infty}} & \lesssim\left\|K\left(t, \partial_{x}\right) \partial_{y} f\right\|_{L_{x}^{\infty} L_{y}^{1}} \\
& \lesssim\left\|K\left(t, \partial_{x}\right) \partial_{y}\langle\nabla\rangle^{\frac{1}{2}+\frac{\epsilon}{2}} f\right\|_{L_{y}^{1} L_{x}^{2}} \\
& =\|\| \widehat{K}(t, \xi) \mathcal{F}_{\xi}\left(\partial_{y}\langle\nabla\rangle^{\frac{1}{2}+\frac{\epsilon}{2}} f\right)(t, \xi, y)\left\|_{L_{\xi}^{2}}\right\|_{L_{y}^{1}} \\
& \lesssim\|\widehat{K}(t, \xi)\|_{L_{\xi}^{\infty}}\|\| \mathcal{F}_{\xi}\left(\partial_{y}\langle\nabla\rangle^{\frac{1}{2}+\frac{\epsilon}{2}} f\right)(t, \xi, y)\left\|_{L_{\xi}^{2}}\right\|_{L_{y}^{1}} \\
& =\|\widehat{K}(t, \xi)\|_{L_{\xi}^{\infty}}\|\| \partial_{y}\langle\nabla\rangle^{\frac{1}{2}+\frac{\epsilon}{2}} f(t, x, y)\left\|_{L_{x}^{2}}\right\|_{L_{y}^{1}} \\
& \lesssim\|\widehat{K}(t, \xi)\|_{L_{\xi}^{\infty}}\left\|\partial_{y}\langle\nabla\rangle^{1+\epsilon} f\right\|_{L_{x y}^{1}} .
\end{aligned}
$$

This proves Lemma 2.4

As a special consequence of Lemmas 2.3 and 2.4, we have the following corollary.

Corollary 2.5. Let $K\left(t, \partial_{x}\right)$ be a Fourier multiplier operator satisfying

$$
\left\|\widehat{\partial_{x}^{\alpha} K}(t, \xi)\right\|_{L_{\xi}^{1}\left(|\xi| \leq \frac{1}{2}\right)}<\infty, \quad\|\widehat{K}(t, \xi)\|_{L_{\xi}^{\infty}\left(|\xi| \geq \frac{1}{2}\right)}<\infty, \quad \alpha \geq 0
$$

Then, for any space-time Schwartz function $f$,

$$
\begin{aligned}
\left\|\partial_{x}^{\alpha} K\left(t, \partial_{x}\right) f\right\|_{L_{x y}^{\infty}} \lesssim & \left(\left\|\widehat{\partial_{x}^{\alpha} K}(t, \xi)\right\|_{L_{\xi}^{1}\left(|\xi| \leq \frac{1}{2}\right)}+\|\widehat{K}(t, \xi)\|_{L_{\xi}^{\infty}\left(|\xi| \geq \frac{1}{2}\right)}\right) \\
& \times\left\|\langle\nabla\rangle^{\alpha+1+\epsilon} \partial_{y} f\right\|_{L_{x y}^{1}} .
\end{aligned}
$$

Lemma 2.6. Assume that $\|\widehat{K}(t, \cdot)\|_{L^{2}}$ is bounded. Then, for any space-time Schwartz function $f$, and any $\epsilon>0$,

$$
\left\|K\left(t, \partial_{x}\right) f\right\|_{L_{x y}^{2}} \lesssim\|\widehat{K}(t, \xi)\|_{L_{\xi}^{2}}\left\||\nabla|^{\frac{1}{2}-\epsilon}\langle\nabla\rangle^{2 \epsilon} f\right\|_{L_{x y}^{1}}
$$

Proof. By a similar manner, we have

$$
\begin{aligned}
\left\|K\left(t, \partial_{x}\right) f\right\|_{L_{x y}^{2}} & =\|\| \widehat{K}(t, \xi) \mathcal{F}_{\xi} f(t, \xi, y)\left\|_{L_{\xi}^{2}}\right\|_{L_{y}^{2}} \\
& \lesssim\|\widehat{K}(t, \xi)\|_{L_{\xi}^{2}}\|\| \mathcal{F}_{\xi} f(t, \xi, y)\left\|_{L_{\xi}^{\infty}}\right\|_{L_{y}^{2}}
\end{aligned}
$$




$$
\begin{aligned}
& \lesssim\|\widehat{K}(t, \xi)\|_{L_{\xi}^{2}}\|\| f(t, x, y)\left\|_{L_{x}^{1}}\right\|_{L_{y}^{2}} \\
& \lesssim\|\widehat{K}(t, \xi)\|_{L_{\xi}^{2}}\|\| f(t, x, y)\left\|_{L_{y}^{2}}\right\|_{L_{x}^{1}} \\
& \lesssim\|\widehat{K}(t, \xi)\|_{L_{\xi}^{2}}\left\||\nabla|^{\frac{1}{2}-\epsilon}\langle\nabla\rangle^{2 \epsilon} f\right\|_{L_{x y}^{1}} .
\end{aligned}
$$

This proves the lemma.

Lemma 2.7. Assume that $\|\widehat{K}(t, \cdot)\|_{L^{\infty}}$ is bounded. Then, for any space-time Schwartz function $f$, and any $\epsilon>0$,

$$
\left\|K\left(t, \partial_{x}\right) f\right\|_{L_{x y}^{2}} \lesssim\|\widehat{K}(t, \xi)\|_{L_{\xi}^{\infty}}\left\||\nabla|^{\frac{1}{2}-\epsilon}\langle\nabla\rangle^{\frac{1}{2}+2 \epsilon} f\right\|_{L_{x y}^{1}}
$$

Proof. By a similar manner, we have

$$
\begin{aligned}
\left\|K\left(t, \partial_{x}\right) f\right\|_{L_{x y}^{2}} & =\|\| \widehat{K}(t, \xi) \mathcal{F}_{\xi} f(t, \xi, y)\left\|_{L_{\xi}^{2}}\right\|_{L_{y}^{2}} \\
& \lesssim\|\widehat{K}(t, \xi)\|_{L_{\xi}^{\infty}}\|\| \mathcal{F}_{\xi} f(t, \xi, y)\left\|_{L_{\xi}^{2}}\right\|_{L_{y}^{2}} \\
& =\|\widehat{K}(t, \xi)\|_{L_{\xi}^{\infty}}\|\| f(t, x, y)\left\|_{L_{x}^{2}}\right\|_{L_{y}^{2}} \\
& \lesssim\|\widehat{K}(t, \xi)\|_{L_{\xi}^{\infty}}\left\||\nabla|^{\frac{1}{2}-\epsilon}\langle\nabla\rangle^{\frac{1}{2}+2 \epsilon} f\right\|_{L_{x y}^{1}}
\end{aligned}
$$

This proves the lemma.

Combining Lemmas 2.6 and 2.7, we have

Corollary 2.8. Assume the Fourier multiplier operator $K\left(t, \partial_{x}\right)$ satisfies

$$
\left\|\widehat{\partial_{x}^{\alpha} K}(t, \xi)\right\|_{L_{\xi}^{2}\left(|\xi| \leq \frac{1}{2}\right)}<\infty, \quad\|\widehat{K}(t, \xi)\|_{L_{\xi}^{\infty}\left(|\xi| \geq \frac{1}{2}\right)}<\infty, \quad \alpha \geq 0 .
$$

Then, for any space-time Schwartz function $f$ and any $\epsilon>0$,

$$
\begin{aligned}
\left\|\partial_{x}^{\alpha} K\left(t, \partial_{x}\right) f\right\|_{L_{x y}^{2}} \lesssim & \left(\left\|\widehat{\partial_{x}^{\alpha} K}(t, \xi)\right\|_{L_{\xi}^{2}\left(|\xi| \leq \frac{1}{2}\right)}+\|\widehat{K}(t, \xi)\|_{L_{\xi}^{\infty}\left(|\xi| \geq \frac{1}{2}\right)}\right) \\
& \cdot\left\|\langle\nabla\rangle^{\alpha+\frac{1}{2}+2 \epsilon}|\nabla|^{\frac{1}{2}-\epsilon} f\right\|_{L_{x y}^{1}} .
\end{aligned}
$$

\section{Proof of Lemma 1.3}

This section provides the proof of Lemma 1.3. More precisely, we show that

$$
\|(\vec{u}, \psi)\|_{Y} \lesssim\|(\vec{u}, \psi)\|_{X}+Q\left(\|(\vec{u}, \psi)\|_{X}\right),
$$

where $X, Y$ and $Q$ are defined as in Introduction.

Proof of Lemma 1.3. First, we recall the basic inequality

$$
\begin{aligned}
\|g(x, y)\|_{L_{x y}^{\infty}} & \lesssim\|g\|_{L^{2}}^{\frac{1}{4}}\left\|\partial_{x} g\right\|_{L^{2}}^{\frac{1}{4}}\left\|\partial_{y} g\right\|_{L^{2}}^{\frac{1}{4}}\left\|\partial_{x} \partial_{y} g\right\|_{L^{2}}^{\frac{1}{4}} \\
& \lesssim\left\|\langle\nabla\rangle \partial_{x} g\right\|_{L_{x y}^{2}}^{\frac{1}{2}}\|\langle\nabla\rangle g\|_{L_{x y}^{2}}^{\frac{1}{2}}
\end{aligned}
$$


We now estimate each term in $Y$ and start with the terms related to $\psi$. By interpolation,

$$
\begin{aligned}
\left\|\langle\nabla\rangle^{3} \partial_{x} \psi(s, x, y)\right\|_{L_{x y}^{2}} & \lesssim\left\|\langle\nabla\rangle^{3} \partial_{x x} \psi\right\|_{L_{x y}^{2}}^{\frac{1}{2}}\left\|\langle\nabla\rangle^{3} \psi\right\|_{L_{x y}^{2}}^{\frac{1}{2}} \\
& \lesssim s^{-\frac{5}{8}}\|(\vec{u}, \psi)\|_{X}^{\frac{1}{2}} s^{-\frac{1}{8}}\|(\vec{u}, \psi)\|_{X}^{\frac{1}{2}} \\
& \lesssim s^{-\frac{3}{4}}\|(\vec{u}, \psi)\|_{X} .
\end{aligned}
$$

By (3.1) and (3.2), we obtain

$$
\left\|\langle\nabla\rangle^{2} \partial_{x} \psi(s, x, y)\right\|_{L_{x y}^{\infty}} \lesssim\left\|\langle\nabla\rangle^{3} \partial_{x x} \psi\right\|_{L_{x y}^{2}}^{\frac{1}{2}}\left\|\langle\nabla\rangle^{3} \partial_{x} \psi\right\|_{L_{x y}^{2}}^{\frac{1}{2}} \lesssim s^{-1}\|(\vec{u}, \psi)\|_{X}
$$

and

$$
\left\|\langle\nabla\rangle^{2} \psi(s, x, y)\right\|_{L_{x y}^{\infty}} \lesssim\left\|\langle\nabla\rangle^{3} \partial_{x} \psi\right\|_{L_{x y}^{2}}^{\frac{1}{2}}\left\|\langle\nabla\rangle^{3} \psi\right\|_{L_{x y}^{2}}^{\frac{1}{2}} \lesssim s^{-\frac{1}{2}}\|(\vec{u}, \psi)\|_{X} .
$$

The other terms in $Y$ are a little tricky. We first construct the following two inequalities, for $j=1,2$ and some $0<\alpha<1$,

$$
\begin{aligned}
\left\|\langle\nabla\rangle \Pi_{j}\right\|_{2} & \lesssim s^{-\frac{5}{4}}\|(\vec{u}, \psi)\|_{Y}^{\alpha}\|(\vec{u}, \psi)\|_{X}^{2-\alpha} \\
\left\|\Pi_{j}\right\|_{\infty} & \lesssim s^{-\frac{3}{2}}\|(\vec{u}, \psi)\|_{Y}^{\alpha}\|(\vec{u}, \psi)\|_{X}^{2-\alpha}
\end{aligned}
$$

Since the cases $j=1$ and $j=2$ can be treated the same way, we only deal with the case $j=1$. According to the expression of $\Pi_{1}$ in (A.1),

$$
\begin{aligned}
\left\|\langle\nabla\rangle \Pi_{1}\right\|_{2} \lesssim & \|\langle\nabla\rangle v\|_{2}\left\|\langle\nabla\rangle \partial_{x} u\right\|_{\infty}+\|\langle\nabla\rangle v\|_{\infty}\left\|\langle\nabla\rangle^{2} u\right\|_{2}+\left\|\langle\nabla\rangle^{3} \psi\right\|_{2}\left\|\langle\nabla\rangle^{2} \partial_{x} \psi\right\|_{\infty} \\
& +\|\langle\nabla\rangle u\|_{2}\left\|\langle\nabla\rangle \partial_{x} u\right\|_{\infty}+\|\langle\nabla\rangle u\|_{2}\left\|\langle\nabla\rangle \partial_{x} v\right\|_{\infty}+\left\|\langle\nabla\rangle \partial_{x x} \psi\right\|_{\infty}\left\|\langle\nabla\rangle^{2} \psi\right\|_{2} \\
= & I_{1}+I_{2}+I_{3}+\text { remainder terms. }
\end{aligned}
$$

The first three terms $I_{1}, I_{2}$ and $I_{3}$ are typical of the terms on the right, and the estimates of remainder terms are similar to them. Therefore, we shall only present their estimates.

$$
\begin{aligned}
I_{1} & =\|\langle\nabla\rangle v\|_{2}\left\|\langle\nabla\rangle \partial_{x} u\right\|_{\infty} \\
& \lesssim\left\|\langle\nabla\rangle^{N} v\right\|_{2}^{\frac{1}{N}}\|v\|_{2}^{1-\frac{1}{N}}\left\|\langle\nabla\rangle \partial_{x} u\right\|_{\infty} \\
& \lesssim s^{\frac{\varepsilon}{N}}\|(\vec{u}, \psi)\|_{X}^{\frac{1}{N}} \cdot s^{-\frac{5}{4}\left(1-\frac{1}{N}\right)}\|(\vec{u}, \psi)\|_{Y}^{1-\frac{1}{N}} \cdot s^{-1}\|(\vec{u}, \psi)\|_{X} \\
& \lesssim s^{-\frac{9}{4}+\frac{5}{4} \cdot \frac{1}{N}+\frac{\varepsilon}{N}}\|(\vec{u}, \psi)\|_{Y}^{1-\frac{1}{N}} \cdot\|(\vec{u}, \psi)\|_{X}^{1+\frac{1}{N}} \\
& \lesssim s^{-\frac{5}{4}}\|(\vec{u}, \psi)\|_{Y}^{1-\frac{1}{N}} \cdot\|(\vec{u}, \psi)\|_{X}^{1+\frac{1}{N}}
\end{aligned}
$$

where $\varepsilon>0$ is a small parameter and $N$ has been chosen big enough.For the second term, by interpolation we obtain

$$
\begin{aligned}
I_{2} & =\|\langle\nabla\rangle v\|_{\infty}\left\|\langle\nabla\rangle^{2} u\right\|_{2} \\
& \lesssim\left\|\langle\nabla\rangle^{N} v\right\|_{2}^{1+\frac{1-\varepsilon}{N-1}}\|v\|_{\infty}^{1-\frac{1-\varepsilon}{N-1}} \\
& \lesssim s^{\frac{1-\varepsilon}{N-1} \cdot \varepsilon}\|(\vec{u}, \psi)\|_{X}^{\frac{1-\varepsilon}{N-1}} s^{-\frac{3}{2}\left(1-\frac{1-\varepsilon}{N-1}\right)}\|(\vec{u}, \psi)\|_{Y}^{1-\frac{1-\varepsilon}{N-1}} s^{\varepsilon}\|(\vec{u}, \psi)\|_{X} \\
& \lesssim s^{-\frac{5}{4}}\|(\vec{u}, \psi)\|_{Y}^{1-\frac{1-\varepsilon}{N-1}} \cdot\|(\vec{u}, \psi)\|_{X}^{1+\frac{1-\varepsilon}{N-1}} .
\end{aligned}
$$


By (3.3), we have

$$
I_{3}=\left\|\langle\nabla\rangle^{3} \psi\right\|_{2}\left\|\langle\nabla\rangle^{2} \partial_{x} \psi\right\|_{\infty} \lesssim s^{-\frac{1}{4}}\|(\vec{u}, \psi)\|_{X} \cdot s^{-1}\|(\vec{u}, \psi)\|_{X} \lesssim s^{-\frac{5}{4}}\|(\vec{u}, \psi)\|_{X}^{2} .
$$

Thus, we have obtained that, for some $\alpha_{1} \in(0,1)$,

$$
\left\|\langle\nabla\rangle \Pi_{1}\right\|_{2} \lesssim s^{-\frac{5}{4}}\|(\vec{u}, \psi)\|_{X}^{2-\alpha_{1}}\|(\vec{u}, \psi)\|_{Y}^{\alpha_{1}}
$$

Through similar computations, for some $\alpha_{2} \in(0,1)$, we can get

$$
\left\|\langle\nabla\rangle \partial_{x} \Pi_{1}\right\|_{2} \lesssim s^{-\frac{7}{4}}\|(\vec{u}, \psi)\|_{X}^{2-\alpha_{2}}\|(\vec{u}, \psi)\|_{Y}^{\alpha_{2}} .
$$

Therefore, by Nash's inequality, we obtain, for some $\alpha \in(0,1)$,

$$
\left\|\Pi_{1}\right\|_{\infty} \lesssim\left\|\langle\nabla\rangle \partial_{x} \Pi_{1}\right\|_{2}^{\frac{1}{2}}\left\|\langle\nabla\rangle \Pi_{1}\right\|_{2}^{\frac{1}{2}} \lesssim s^{-\frac{3}{2}}\|(\vec{u}, \psi)\|_{X}^{2-\alpha}\|(\vec{u}, \psi)\|_{Y}^{\alpha}
$$

Thus we complete the proof of (3.5) and (3.6).

Now, using the inequalities (3.2), (3.3), (3.5), (3.6) with $j=1$ and (1.5), we have for small $\eta_{0}>0, s \geq 1$,

$$
\begin{aligned}
\|u\|_{\infty} & \lesssim\left\|\partial_{t} u\right\|_{\infty}+\left\|\langle\nabla\rangle \partial_{x} \psi\right\|_{\infty}+\left\|\Pi_{1}\right\|_{\infty} \\
& \lesssim s^{-\frac{3}{2}}\|(\vec{u}, \psi)\|_{X}+s^{-1}\|(\vec{u}, \psi)\|_{X}+s^{-\frac{3}{2}}\|(\vec{u}, \psi)\|_{Y}^{\alpha}\|(\vec{u}, \psi)\|_{X}^{2-\alpha} \\
& \lesssim s^{-1}\left(\|(\vec{u}, \psi)\|_{X}+C\left(\eta_{0}\right)\|(\vec{u}, \psi)\|_{X}^{2}\right)+s^{-1} \eta_{0}\|(\vec{u}, \psi)\|_{Y} \\
\|u\|_{2} & \lesssim\left\|\partial_{t} u\right\|_{2}+\left\|\langle\nabla\rangle \partial_{x} \psi\right\|_{2}+\left\|\Pi_{1}\right\|_{2} \\
& \lesssim s^{-\frac{5}{4}}\|(\vec{u}, \psi)\|_{X}+s^{-\frac{3}{4}}\|(\vec{u}, \psi)\|_{X}+s^{-\frac{5}{4}}\|(\vec{u}, \psi)\|_{Y}^{\alpha}\|(\vec{u}, \psi)\|_{X}^{2-\alpha} \\
& \lesssim s^{-\frac{3}{4}}\left(\|(\vec{u}, \psi)\|_{X}+C\left(\eta_{0}\right)\|(\vec{u}, \psi)\|_{X}^{2}\right)+s^{-\frac{3}{4}} \eta_{0}\|(\vec{u}, \psi)\|_{Y} . \\
\left\|\partial_{x} u\right\|_{2} & \lesssim\left\|\partial_{x} \partial_{t} u\right\|_{2}+\left\|\langle\nabla\rangle \partial_{x x} \psi\right\|_{2}+\left\|\partial_{x} \Pi_{1}\right\|_{2} \\
& \lesssim\left\|\langle\nabla\rangle \partial_{t} u\right\|_{2}+\left\|\langle\nabla\rangle \partial_{x x} \psi\right\|_{2}+\left\|\langle\nabla\rangle \Pi_{1}\right\|_{2} \\
& \lesssim\left\|\langle\nabla\rangle \partial_{t} u\right\|_{2}+\left\|\langle\nabla\rangle \partial_{x x} \psi\right\|_{2}+s^{-\frac{5}{4}}\|(\vec{u}, \psi)\|_{Y}^{\alpha}\|(\vec{u}, \psi)\|_{X}^{2-\alpha} \\
& \lesssim s^{-\frac{5}{4}}\left(\|(\vec{u}, \psi)\|\left\|_{X}+C\left(\eta_{0}\right)\right\|(\vec{u}, \psi) \|_{X}^{2}\right)+s^{-\frac{5}{4}} \eta_{0}\|(\vec{u}, \psi)\|_{Y} .
\end{aligned}
$$

Similarly, by equation (1.6) and inequalities (3.3), (3.5) and (3.6) with $j=2$, we have

$$
\begin{aligned}
\|v\|_{\infty} & \lesssim s^{-\frac{3}{2}}\left(\|(\vec{u}, \psi)\|_{X}+C\left(\eta_{0}\right)\|(\vec{u}, \psi)\|_{X}^{2}\right)+s^{-\frac{3}{2}} \eta_{0}\|(\vec{u}, \psi)\|_{Y} . \\
\|v\|_{2} & \lesssim s^{-\frac{5}{4}}\left(\|(\vec{u}, \psi)\|_{X}+C\left(\eta_{0}\right)\|(\vec{u}, \psi)\|_{X}^{2}\right)+s^{-\frac{5}{4}} \eta_{0}\|(\vec{u}, \psi)\|_{Y} .
\end{aligned}
$$

Therefore, collecting the estimates above, we prove that

$$
\|(\vec{u}, \psi)\|_{Y} \lesssim\|(\vec{u}, \psi)\|_{X}+\eta_{0}\|(\vec{u}, \psi)\|_{Y}+\|(\vec{u}, \psi)\|_{X}^{2} .
$$

Choosing $\eta_{0}$ small enough, we get

$$
\|(\vec{u}, \psi)\|_{Y} \lesssim\|(\vec{u}, \psi)\|_{X}+\|(\vec{u}, \psi)\|_{X}^{2} .
$$

This completes the proof of Lemma 1.3. 


\section{EnERgy estimates}

The rest of the sections are devoted to proving (1.17), namely

$$
\|(\vec{u}, \psi)\|_{X} \lesssim\left\|\left(\vec{u}_{0}, \psi_{0}\right)\right\|_{X_{0}}+Q\left(\|(\vec{u}, \psi)\|_{Y}\right) .
$$

This section shows that the first term in the definition of the norm of $X$ obeys this inequality.

For this purpose, we first show that, for any real number $\sigma>0$,

$$
\begin{aligned}
& \frac{d}{d t}\left(\left\|\langle\nabla\rangle^{\sigma} \vec{u}\right\|_{L^{2}}^{2}+\left\|\langle\nabla\rangle^{\sigma} \vec{b}\right\|_{L^{2}}^{2}\right) \\
\lesssim & \left(\|\nabla \vec{u}\|_{L^{\infty}}+\|\nabla \vec{b}\|_{L^{\infty}}^{2}\right)\left(\left\|\langle\nabla\rangle^{\sigma} \vec{u}\right\|_{L^{2}}^{2}+\left\|\langle\nabla\rangle^{\sigma} \vec{b}\right\|_{L^{2}}^{2}\right) .
\end{aligned}
$$

To do so, we take advantage of (1.2), which is equivalent to (1.1). Applying $\langle\nabla\rangle^{\sigma}$ to (1.2) and taking the inner product with $\left(\langle\nabla\rangle^{\sigma} \vec{u},\langle\nabla\rangle^{\sigma} \vec{b}\right)$, we obtain, after integrating by parts and invoking $\nabla \cdot \vec{u}=0$,

$$
\begin{aligned}
\frac{1}{2} \frac{d}{d t} & \left(\left\|\langle\nabla\rangle^{\sigma} \vec{u}\right\|_{L^{2}}^{2}+\left\|\langle\nabla\rangle^{\sigma} \vec{b}\right\|_{L^{2}}^{2}\right)+\left\|\langle\nabla\rangle^{\sigma} \vec{u}\right\|_{L^{2}}^{2} \\
& =-\int\left[\langle\nabla\rangle^{\sigma}, \vec{u} \cdot \nabla\right] \vec{u} \cdot\langle\nabla\rangle^{\sigma} \vec{u} d x-\int\left[\langle\nabla\rangle^{\sigma}, \vec{u} \cdot \nabla\right] \vec{b} \cdot\langle\nabla\rangle^{\sigma} \vec{b} d x \\
& +\int\left[\langle\nabla\rangle^{\sigma}, \vec{b} \cdot \nabla\right] \vec{b} \cdot\langle\nabla\rangle^{\sigma} \vec{u} d x+\int\left[\langle\nabla\rangle^{\sigma}, \vec{b} \cdot \nabla\right] \vec{u} \cdot\langle\nabla\rangle^{\sigma} \vec{b} d x,
\end{aligned}
$$

where we have used the standard commutator notation

$$
\left[\langle\nabla\rangle^{\sigma}, \vec{u} \cdot \nabla\right] \vec{u}=\langle\nabla\rangle^{\sigma}(\vec{u} \cdot \nabla \vec{u})-\vec{u} \cdot \nabla\langle\nabla\rangle^{\sigma} \vec{u} .
$$

By Hölder's inequality and a standard commutator estimate, we have

$$
\begin{aligned}
\left|\int\left[\langle\nabla\rangle^{\sigma}, \vec{u} \cdot \nabla\right] \vec{u} \cdot\langle\nabla\rangle^{\sigma} \vec{u} d x\right| & \leq\left\|\left[\langle\nabla\rangle^{\sigma}, \vec{u} \cdot \nabla\right] \vec{u}\right\|_{L^{2}}\left\|\langle\nabla\rangle^{\sigma} \vec{u}\right\|_{L^{2}} \\
& \lesssim\left\|\langle\nabla\rangle^{\sigma} \vec{u}\right\|_{L^{2}}\|\nabla \vec{u}\|_{L^{\infty}}\left\|\langle\nabla\rangle^{\sigma} \vec{u}\right\|_{L^{2}},
\end{aligned}
$$

moreover, for some constant $C>0$,

$$
\begin{aligned}
\left|\int\left[\langle\nabla\rangle^{\sigma}, \vec{b} \cdot \nabla\right] \vec{u} \cdot\langle\nabla\rangle^{\sigma} \vec{b} d x\right| \leq\left\|\left[\langle\nabla\rangle^{\sigma}, \vec{b} \cdot \nabla\right] \vec{u}\right\|_{L^{2}}\left\|\langle\nabla\rangle^{\sigma} \vec{b}\right\|_{L^{2}} \\
\quad \lesssim\left(\left\|\langle\nabla\rangle^{\sigma} \vec{u}\right\|_{L^{2}}\|\nabla \vec{b}\|_{L^{\infty}}+\left\|\langle\nabla\rangle^{\sigma} \vec{b}\right\|_{L^{2}}\|\nabla \vec{u}\|_{L^{\infty}}\right)\left\|\langle\nabla\rangle^{\sigma} \vec{b}\right\|_{L^{2}} \\
\leq \frac{1}{4}\left\|\langle\nabla\rangle^{\sigma} \vec{u}\right\|_{L^{2}}^{2}+C\left(\|\nabla \vec{b}\|_{L^{\infty}}^{2}+\|\nabla \vec{u}\|_{L^{\infty}}\right)\left\|\langle\nabla\rangle^{\sigma} \vec{b}\right\|_{L^{2}}^{2} .
\end{aligned}
$$

The other two terms can be similarly bounded. We obtain (4.1) after we insert the estimates above in (4.2). Setting $\sigma=N$ and integrating (4.1) in time, we obtain

$$
\left\|\langle\nabla\rangle^{N}(\vec{u}, \vec{b})\right\|_{L^{2}}^{2} \lesssim\left\|\left(\vec{u}_{0}, \psi_{0}\right)\right\|_{X_{0}}^{2}+\int_{1}^{t}\left(\|\nabla \vec{u}\|_{L^{\infty}}+\|\nabla \vec{b}\|_{L^{\infty}}^{2}\right) \cdot\left\|\langle\nabla\rangle^{N}(\vec{u}, \vec{b})\right\|_{L^{2}}^{2} d s .
$$

To bound $\|\nabla \vec{b}\|_{L^{\infty}}$, by the definition of $Y$,

$$
\|\nabla \vec{b}(s)\|_{L^{\infty}}^{2} \lesssim\left\|\langle\nabla\rangle^{2} \psi(s)\right\|_{L^{\infty}}^{2} \lesssim s^{-1}\|(\vec{u}, \psi)\|_{Y}^{2} .
$$


Also, we have

$$
\|\nabla \vec{u}(s)\|_{L^{\infty}} \leq\|\nabla u(s)\|_{L^{\infty}}+\|\nabla v(s)\|_{L^{\infty}} \leq s^{-1}\|(\vec{u}, \psi)\|_{Y} .
$$

Therefore,

$$
\begin{aligned}
\left\|\langle\nabla\rangle^{N}(\vec{u}, \vec{b})\right\|_{L^{2}}^{2} & \lesssim\left\|\left(\vec{u}_{0}, \psi_{0}\right)\right\|_{X_{0}}^{2}+\int_{1}^{t}\langle s\rangle^{-1+2 \varepsilon} d s\left(\|(\vec{u}, \psi)\|_{Y}+\|(\vec{u}, \psi)\|_{Y}^{2}\right)\|(\vec{u}, \psi)\|_{Y}^{2} \\
& \lesssim\left\|\left(\vec{u}_{0}, \psi_{0}\right)\right\|_{X_{0}}^{2}+t^{2 \varepsilon}\left(\|(\vec{u}, \psi)\|_{Y}^{3}+\|(\vec{u}, \psi)\|_{Y}^{4}\right) .
\end{aligned}
$$

This proves that

$$
\sup _{t \geq 1}\left(t^{-\varepsilon}\left\|\langle\nabla\rangle^{N}(\vec{u}(t), \nabla \psi(t))\right\|_{2}\right) \lesssim\left\|\left(\vec{u}_{0}, \psi_{0}\right)\right\|_{X_{0}}+\left(\|(\vec{u}, \psi)\|_{Y}^{3}+\|(\vec{u}, \psi)\|_{Y}^{4}\right)^{\frac{1}{2}}
$$

\section{Estimates ON NONLINEARITIES}

This section estimates the nonlinear terms $F_{0}, F_{1}, F_{2}$, defined in (1.10). These bounds will be used in the proof of (1.17) given in the subsequent sections.

We will use the Littlewood-Paley projection operators. Let $\phi(\xi)$ be a smooth bump function supported in the ball $|\xi| \leq 2$ and equals one on the ball $|\xi| \leq 1$. For any real number $M>0$ and $f \in \mathcal{S}^{\prime}$ (tempered distributions), the projection operators can be defined as follows:

$$
\begin{aligned}
& \widehat{P_{\leq M} f}(\xi):=\phi(\xi / M) \hat{f}(\xi), \\
& \widehat{P_{>M} f}(\xi):=(1-\phi(\xi / M)) \hat{f}(\xi), \\
& \widehat{P_{M} f}(\xi):=(\phi(\xi / M)-\phi(2 \xi / M)) \hat{f}(\xi) .
\end{aligned}
$$

We also need the following estimate involving the Riesz transform $\mathcal{R}$.

Lemma 5.1. For any $\epsilon>0$,

$$
\|\mathcal{R} f\|_{L_{x y}^{1}} \lesssim\left\||\nabla|^{-\epsilon}\langle\nabla\rangle^{2 \epsilon} f\right\|_{L_{x y}^{1}} .
$$

The proof of Lemma 5.1 is presented in Appendix A.2.

Lemma 5.2. For any $s \geq 1$,

$$
\left\|\langle\nabla\rangle^{5}|\nabla|^{\frac{1}{2}-\epsilon} F_{1}(s, \cdot)\right\|_{L_{x y}^{1}} \lesssim s^{-\frac{3}{2}-\varepsilon} Q\left(\|(\vec{u}, \psi)\|_{Y}\right),
$$

where $\epsilon$ is same as in Corollary [2.8, and $\varepsilon$ is same as in (1.15).

Proof. By definition (1.12),

$$
F_{1}=-\partial_{x} \partial_{y}\left(u \partial_{x} \psi\right)-\partial_{x} \partial_{y}\left(v \partial_{y} \psi\right)+\partial_{t} \Pi_{1},
$$

where $\Pi_{1}$ is explicitly given in Appendix A.1. Since $F_{1}$ is a quadratic nonlinearity, we write

$$
F_{1}=F_{11}(u, v)+F_{12}(u, \psi)+F_{13}(v, \psi)+F_{14}(u, u)+F_{15}(v, v)+F_{16}(\psi, \psi),
$$

where $F_{11}(u, v)$ is a collection of the terms which contain the unknown function $(u, v)$, and $F_{12}, \cdots, F_{16}$ are defined similarly. To bound these terms, we split $F_{1}$ into low 
frequency parts and high frequency parts. More precisely, for small $\delta>0$ to be specified later, we write

$$
F_{1}:=F_{1, \text { high }}+F_{1, \text { low }}
$$

where

$$
\begin{aligned}
& F_{1, \text { high }}=F_{11}\left(\left(1-P_{\leq s^{\delta}}\right) u, v\right)+F_{11}\left(P_{\leq s^{\delta}} u,\left(1-P_{\leq s^{\delta}}\right) v\right)+\cdots, \\
& F_{1, \text { low }}=F_{11}\left(P_{\leq s^{\delta}} u, P_{\leq s^{\delta}} v\right)+\cdots,
\end{aligned}
$$

that is, each term in $F_{1, \text { high }}$ contains at least one high frequency part and the terms in $F_{1, \text { low }}$ involve only low frequencies.

Although the number of the terms in $F_{1}$ is large, they can be treated similarly. For the sake of clarity, we shall only present the estimates for a representative term. That is, we write

$$
F_{1}=\frac{\partial_{y} \partial_{y}}{\Delta}\left(\partial_{y} \psi \partial_{x} \partial_{y} \psi_{t}\right)+\text { similar terms }
$$

We now focus on the representative term $\frac{\partial_{y} \partial_{y}}{\Delta}\left(\partial_{y} \psi \partial_{x} \partial_{y} \psi_{t}\right)$. As in (5.4), we split it into high and low frequency terms. First, we deal with those involving high frequencies, which can be treated by a standard way (see [19] for some related analysis). We focus on $\frac{\partial_{x} \partial_{y}}{\Delta}\left(\partial_{y} \psi \partial_{x} \partial_{y} P_{\gtrsim_{s} \delta} \psi_{t}\right)$ and by (5.1),

$$
\begin{aligned}
& \left\|\langle\nabla\rangle^{5}|\nabla|^{\frac{1}{2}-\epsilon} \frac{\partial_{y} \partial_{y}}{\Delta}\left(\partial_{y} \psi \partial_{x} \partial_{y} P_{\gtrsim s^{\delta}} \psi_{t}\right)\right\|_{L_{x y}^{1}} \\
& \lesssim\left\|\langle\nabla\rangle^{5+2 \epsilon}|\nabla|^{\frac{1}{2}-2 \epsilon}\left(\partial_{y} \psi \partial_{x} \partial_{y} P_{\gtrsim s^{\delta}} \psi_{t}\right)\right\|_{L_{x y}^{1}} \\
& \lesssim\left\|\langle\nabla\rangle^{6}\left(\partial_{y} \psi \partial_{x} \partial_{y} P_{\gtrsim s^{\delta}} \psi_{t}\right)\right\|_{L_{x y}^{1}} \\
& \lesssim\left\|\langle\nabla\rangle^{6} \partial_{y} \psi\right\|_{L_{x y}^{2}}\left\|\partial_{x} \partial_{y} P_{\gtrsim^{\delta}} \psi_{t}\right\|_{L_{x y}^{2}}+\left\|\partial_{y} \psi\right\|_{L_{x y}^{2}}\left\|\langle\nabla\rangle^{6} \partial_{x} \partial_{y} P_{\gtrsim^{\delta}} \psi_{t}\right\|_{L_{x y}^{2}} \\
& \lesssim\left\|\langle\nabla\rangle^{7} \psi\right\|_{L_{x y}^{2}}\left\|\langle\nabla\rangle^{8} P_{\gtrsim^{s}} \psi_{t}\right\|_{L_{x y}^{2}} \text {. }
\end{aligned}
$$

Since $\psi_{t}=-v-\vec{u} \cdot \nabla \psi$, we have, for the enough large $N$,

$$
\begin{aligned}
\left\|\langle\nabla\rangle^{8} P_{\gtrsim s^{\delta}} \psi_{t}\right\|_{L_{x y}^{2}} & \lesssim\left\|\langle\nabla\rangle^{8} P_{\gtrsim s^{\delta}} v\right\|_{L_{x y}^{2}}+\left\|\langle\nabla\rangle^{8} P_{\gtrsim s^{\delta}}(\vec{u} \cdot \nabla \psi)\right\|_{L_{x y}^{2}} \\
& \lesssim s^{-(N-8) \delta}\left(\left\|\langle\nabla\rangle^{N} v\right\|_{L_{x y}^{2}}+\left\|\langle\nabla\rangle^{N}(\vec{u} \cdot \nabla \psi)\right\|_{L_{x y}^{2}}\right) \\
& \lesssim s^{-(N-8) \delta}\left(\left\|\langle\nabla\rangle^{N} v\right\|_{L_{x y}^{2}}+\left\|\langle\nabla\rangle^{N} \vec{u}\right\|_{L_{x y}^{2}} \cdot\left\|\langle\nabla\rangle^{N+1} \psi\right\|_{L_{x y}^{2}}\right) \\
& \lesssim s^{-\frac{3}{2}-2 \varepsilon}\left(\|(\vec{u}, \psi)\|_{Y}+\|(\vec{u}, \psi)\|_{Y}^{2}\right) .
\end{aligned}
$$

Therefore, combining with above two estimates, we obtain that

$$
\left\|\langle\nabla\rangle^{5}|\nabla|^{\frac{1}{2}-\epsilon} \frac{\partial_{y} \partial_{y}}{\Delta}\left(\partial_{y} \psi \partial_{x} \partial_{y} P_{\gtrsim^{\delta}} \delta \psi_{t}\right)\right\|_{L_{x y}^{1}} \lesssim s^{-\frac{3}{2}-\varepsilon} Q\left(\|(\vec{u}, \psi)\|_{Y}\right) .
$$


Now we turn to $F_{1, l o w}$. Again, using (5.1), we have

$$
\begin{aligned}
&\left\|\langle\nabla\rangle^{5}|\nabla|^{\frac{1}{2}-\epsilon} \frac{\partial_{y} \partial_{y}}{\Delta}\left(\partial_{y} P_{\leq s^{\delta}} \psi \partial_{x} \partial_{y} P_{\leq s^{\delta}} \psi_{t}\right)\right\|_{L_{x y}^{1}} \\
&=\left\|\langle\nabla\rangle^{5}|\nabla|^{\frac{1}{2}-\epsilon} \frac{\partial_{y} \partial_{y}}{\Delta} P_{\leq 4 s^{\delta}}\left(\partial_{y} P_{\leq s^{\delta}} \psi \partial_{x} \partial_{y} P_{\leq s^{\delta}} \psi_{t}\right)\right\|_{L_{x y}^{1}} \\
& \lesssim\left\|\langle\nabla\rangle^{5+2 \epsilon}|\nabla|^{\frac{1}{2}-2 \epsilon} P_{\leq 4 s^{\delta}}\left(\partial_{y} P_{\leq s^{\delta}} \psi \partial_{x} \partial_{y} P_{\leq s^{\delta}} \psi_{t}\right)\right\|_{L_{x y}^{1}} \\
& \lesssim s^{6 \delta}\left\|\partial_{y} P_{\leq s^{\delta}} \psi \partial_{x} \partial_{y} P_{\leq s^{\delta}} \psi_{t}\right\|_{L_{x y}^{1}} \lesssim s^{6 \delta}\left\|\partial_{y} P_{\leq s^{\delta}} \psi\right\|_{L_{x y}^{2}}\left\|\partial_{x} \partial_{y} P_{\leq s^{\delta}} \psi_{t}\right\|_{L_{x y}^{2}} \\
& \lesssim s^{8 \delta}\|\psi\|_{L_{x y}^{2}}\left\|\partial_{x} \psi_{t}\right\|_{L_{x y}^{2}} .
\end{aligned}
$$

Now we need the following estimate,

$$
\left\|\partial_{x} \psi_{t}\right\|_{L_{x y}^{2}} \lesssim s^{-\frac{3}{2}}\left(\|(\vec{u}, \psi)\|_{Y}+Q\left(\|(\vec{u}, \psi)\|_{Y}\right)\right) .
$$

Indeed, by equations (1.6) and (1.7), we have

$$
\begin{aligned}
\left\|\partial_{x} \psi_{t}\right\|_{L_{x y}^{2}} & \lesssim\left\|\partial_{x} v\right\|_{2}+\left\|\partial_{x}(\vec{u} \cdot \nabla \psi)\right\|_{2} \\
& \lesssim\left\|\partial_{x} \partial_{t} v\right\|_{2}+\left\|\partial_{x}^{3} \psi\right\|_{2}+\left(\left\|\partial_{x} u\right\|_{2}+\left\|\partial_{x} v\right\|_{2}\right)\|\langle\nabla\rangle \psi\|_{\infty} \\
& +\left(\|u\|_{\infty}+\|v\|_{\infty}\right)\left\|\langle\nabla\rangle \partial_{x} \psi\right\|_{2}+\left\|\partial_{x} \Pi_{2}\right\|_{2}
\end{aligned}
$$

As in the proof of (3.6), we have

$$
\left\|\partial_{x} \Pi_{2}\right\|_{2} \lesssim s^{-\frac{3}{2}} Q\left(\|(\vec{u}, \psi)\|_{Y}\right) .
$$

Then, (5.6) follows from (5.7). By (5.6), for any $8 \delta \leq \frac{1}{4}+\varepsilon$, we get

$$
\begin{aligned}
\left\|\langle\nabla\rangle^{5}|\nabla|^{\frac{1}{2}-\epsilon} \frac{\partial_{y} \partial_{y}}{-\Delta}\left(\partial_{y} P_{\leq s^{\delta}} \psi \partial_{x} \partial_{y} P_{\leq s^{\delta}} \psi_{t}\right)\right\|_{L_{x y}^{1}} & \lesssim s^{8 \delta} s^{-\frac{7}{4}}\|(\vec{u}, \psi)\|_{Y}^{2} \\
& \lesssim s^{-\frac{3}{2}-\varepsilon} Q\left(\|(\vec{u}, \psi)\|_{Y}\right) .
\end{aligned}
$$

This proves the lemma.

Lemma 5.3. Let $\epsilon, \varepsilon$ be the same as in Lemma 5.2. Then for any $s \geq 1$,

$$
\left\|\langle\nabla\rangle^{5}|\nabla|^{\frac{1}{2}-\epsilon} F_{2}\right\|_{L_{x y}^{1}} \lesssim s^{-\frac{3}{2}-\varepsilon} Q\left(\|(\vec{u}, \psi)\|_{Y}\right) .
$$

Proof. As before, we write

$$
\begin{aligned}
F_{2} & =\partial_{t} \Pi_{2}+\partial_{x} \partial_{x}(\vec{u} \nabla \psi) \\
& =-2 \frac{\partial_{x} \partial_{y}}{\Delta}\left(\partial_{y} \psi \partial_{x} \partial_{y} \psi_{t}\right)+\text { similar terms }
\end{aligned}
$$

Since (5.9) has the similar form as (5.3), we have the same estimate as the one for $F_{1}$. The details are omitted here.

The nonlinear term $F_{0}$ behaves much differently from $F_{1}, F_{2}$, indeed, we have 
Lemma 5.4. Let $\epsilon, \varepsilon$ be the same as in Lemma 5.2. Then for any $s \geq 1$,

$$
\begin{gathered}
\left\|\langle\nabla\rangle^{5}|\nabla|^{\frac{1}{2}-\epsilon} F_{0}\right\|_{L_{x y}^{1}} \lesssim s^{-1-\varepsilon} Q\left(\|(\vec{u}, \psi)\|_{Y}\right) ; \\
\left\|\langle\nabla\rangle^{\frac{11}{2}+2 \epsilon} \nabla F_{0}\right\|_{L_{x y}^{1}} \lesssim s^{-\frac{3}{2}+\varepsilon} Q\left(\|(\vec{u}, \psi)\|_{Y}\right) ; \\
\left\|\langle\nabla\rangle^{5} \nabla \partial_{x} F_{0}\right\|_{L_{x y}^{1}} \lesssim s^{-\frac{3}{2}-\varepsilon} Q\left(\|(\vec{u}, \psi)\|_{Y}\right) .
\end{gathered}
$$

Proof. By (1.11) we write

$$
\begin{aligned}
F_{0} & =-\left(\vec{u}+\vec{u}_{t}\right) \cdot \nabla \phi-\Pi_{2} \\
& =-\Pi_{2}-\partial_{x} \partial_{y} \psi \partial_{x} \psi-\Pi_{1} \partial_{x} \psi+\partial_{x}^{2} \psi \partial_{y} \psi-\Pi_{2} \partial_{y} \psi-u \partial_{x} \psi_{t}-v \partial_{y} \psi_{t} \\
& =-\frac{\partial_{x} \partial_{y}}{\Delta}\left(\partial_{y} \psi \partial_{x} \partial_{y} \psi\right)+\text { similar terms. }
\end{aligned}
$$

We also write

where

$$
F_{0}:=F_{0, l o w}+F_{0, h i g h},
$$

$$
F_{0, \text { low }}=F_{0}\left(P_{\leq s^{\delta}} u, P_{\leq s^{\delta}} v, P_{\leq s^{\delta}} \psi\right) .
$$

We only consider $F_{0, \text { low }}$, since the piece $F_{0, \text { high }}$ can be treated in the manner as in the proof of Lemma 5.2. We need the following lemma.

Lemma 5.5. Let $\alpha>0, N_{0} \geq 0$, then for any $\epsilon>0$,

$$
\left\||\nabla|^{\alpha}\langle\nabla\rangle^{N_{0}} \frac{\partial_{x} \partial_{y}}{-\Delta} f\right\|_{L_{x y}^{1}} \lesssim\|f\|_{L_{x y}^{1}}^{1-\alpha+\epsilon}\left\|\partial_{x} f\right\|_{L_{x y}^{1}}^{\alpha-\epsilon}+\left\|\langle\nabla\rangle^{N_{0}-1+\alpha+\epsilon} \partial_{x} f\right\|_{L_{x y}^{1}} .
$$

The proof of Lemma 5.5 is presented in Appendix A.3.

To prove (5.10), we use Lemma 5.5 for $\alpha=\frac{1}{2}-\epsilon$ to get

$$
\begin{aligned}
& \left\|\langle\nabla\rangle^{\frac{11}{2}+2 \epsilon}|\nabla|^{\frac{1}{2}-\epsilon} \frac{\partial_{x} \partial_{y}}{-\Delta}\left(\partial_{y} P_{\leq s^{\delta}} \psi \partial_{x} \partial_{y} P_{\leq s^{\delta}} \psi\right)\right\|_{L_{x y}^{1}} \\
\lesssim & \left\|\partial_{y} P_{\leq s^{\delta}} \psi \partial_{x} \partial_{y} P_{\leq s^{\delta}} \psi\right\|_{L_{x y}^{1}}^{\frac{1}{2}+2 \epsilon}\left\|\partial_{x}\left(\partial_{y} P_{\leq s^{\delta}} \psi \partial_{x} \partial_{y} P_{\leq s^{\delta}} \psi\right)\right\|_{L_{x y}^{1}}^{\frac{1}{2}-2 \epsilon} \\
& +\left\|\langle\nabla\rangle^{6+2 \epsilon} \partial_{x}\left(\partial_{y} P_{\leq s^{\delta}} \psi \partial_{x} \partial_{y} P_{\leq s^{\delta}} \psi\right)\right\|_{L_{x y}^{1}} \cdot
\end{aligned}
$$

By Hölder's inequality, we have

$$
\begin{aligned}
& \quad\left\|\partial_{y} P_{\leq s^{\delta}} \psi \partial_{x} \partial_{y} P_{\leq s^{\delta}} \psi\right\|_{L_{x y}^{1}} \lesssim\left\|\partial_{y} P_{\leq s^{\delta}} \psi\right\|_{L_{x y}^{2}}\left\|\partial_{x} \partial_{y} P_{\leq s^{\delta}} \psi\right\|_{L_{x y}^{2}} \\
& \lesssim s^{2 \delta}\|\psi\|_{L_{x y}^{2}}\left\|\partial_{x} \psi\right\|_{L_{x y}^{2}} \lesssim s^{2 \delta-1}\|(\vec{u}, \psi)\|_{Y}^{2} .
\end{aligned}
$$

Moreover,

$$
\begin{aligned}
& \left\|\partial_{x}\left(\partial_{y} P_{\leq s^{\delta}} \psi \partial_{x} \partial_{y} P_{\leq s^{\delta}} \psi\right)\right\|_{L_{x y}^{1}} \\
\lesssim & \left\|\partial_{x} \partial_{y} P_{\leq s^{\delta}} \psi\right\|_{L_{x y}^{2}}^{2}+\left\|\partial_{y} P_{\leq s^{\delta}} \psi\right\|_{L_{x y}^{2}}\left\|\partial_{x}^{2} \partial_{y} P_{\leq s^{\delta}} \psi\right\|_{L_{x y}^{2}} \\
\lesssim & s^{2 \delta}\left\|\partial_{x} \psi\right\|_{L_{x y}^{2}}^{2}+s^{2 \delta}\|\psi\|_{L_{x y}^{2}}\left\|\partial_{x}^{2} \psi\right\|_{L_{x y}^{2}} \\
\lesssim & s^{2 \delta-\frac{3}{2}}\|(\vec{u}, \psi)\|_{Y}^{2}
\end{aligned}
$$


and using (5.16),

$$
\begin{aligned}
\left\|\langle\nabla\rangle^{6+2 \epsilon} \partial_{x}\left(\partial_{y} P_{\leq s^{\delta}} \psi \partial_{x} \partial_{y} P_{\leq s^{\delta}} \psi\right)\right\|_{L_{x y}^{1}} & \lesssim s^{(6+2 \epsilon) \delta}\left\|\partial_{x}\left(\partial_{y} P_{\leq s^{\delta}} \psi \partial_{x} \partial_{y} P_{\leq s^{\delta}} \psi\right)\right\|_{L_{x y}^{1}} \\
& \lesssim s^{9 \delta-\frac{3}{2}}\|(\vec{u}, \psi)\|_{Y}^{2} .
\end{aligned}
$$

Therefore, combining (5.15)-(5.17), we deduce that, for any $\delta>0$ satisfying $9 \delta+\varepsilon \leq \frac{1}{2}$,

$$
\left\|\langle\nabla\rangle^{5}|\nabla|^{\frac{1}{2}-\epsilon} \frac{\partial_{x} \partial_{y}}{-\Delta}\left(\partial_{y} P_{\leq s^{\delta}} \psi \partial_{x} \partial_{y} P_{\leq s^{\delta}} \psi\right)\right\|_{L_{x y}^{1}} \lesssim s^{-1-\varepsilon}\|(\vec{u}, \psi)\|_{Y}^{2} .
$$

For (5.11), we only need to prove

$$
\left\|\langle\nabla\rangle^{5} \nabla \frac{\partial_{x} \partial_{y}}{-\Delta}\left(\partial_{y} P_{\leq s^{\delta}} \psi \partial_{x} \partial_{y} P_{\leq s^{\delta}} \psi\right)\right\|_{L_{x y}^{1}} \lesssim s^{-\frac{3}{2}+\varepsilon} Q\left(\|(\vec{u}, \psi)\|_{Y}\right) .
$$

By Lemma 5.1 and Lemma 5.5 for $\alpha=1-\epsilon$, and by (5.15)-(5.17) we have

$$
\begin{aligned}
&\left\|\langle\nabla\rangle^{5} \nabla \frac{\partial_{x} \partial_{y}}{-\Delta}\left(\partial_{y} P_{\leq s^{\delta}} \psi \partial_{x} \partial_{y} P_{\leq s^{\delta}} \psi\right)\right\|_{L_{x y}^{1}} \\
& \lesssim\left\|\langle\nabla\rangle^{5+2 \epsilon}|\nabla|^{1-\epsilon} \frac{\partial_{x} \partial_{y}}{-\Delta}\left(\partial_{y} P_{\leq s^{\delta}} \psi \partial_{x} \partial_{y} P_{\leq s^{\delta}} \psi\right)\right\|_{L_{x y}^{1}} \\
& \lesssim\left\|\partial_{y} P_{\leq s^{\delta}} \psi \partial_{x} \partial_{y} P_{\leq s^{\delta}} \psi\right\|_{L_{x y}^{1}}^{2 \epsilon}\left\|\partial_{x}\left(\partial_{y} P_{\leq s^{\delta}} \psi \partial_{x} \partial_{y} P_{\leq s^{\delta}} \psi\right)\right\|_{L_{x y}^{1}}^{1-2 \epsilon} \\
&+\left\|\langle\nabla\rangle^{6+2 \epsilon} \partial_{x}\left(\partial_{y} P_{\leq s^{\delta}} \psi \partial_{x} \partial_{y} P_{\leq s^{\delta}} \psi\right)\right\|_{L_{x y}^{1}} \\
& \lesssim\left(s^{2 \epsilon(2 \delta-1)+(1-2 \epsilon)\left(2 \delta-\frac{3}{2}\right)}+s^{9 \delta-\frac{3}{2}}\right)\|(\vec{u}, \psi)\|_{Y}^{2} \\
& \lesssim s^{-\frac{3}{2}+\varepsilon}\|(\vec{u}, \psi)\|_{Y}^{2}
\end{aligned}
$$

This gives (5.11). To prove (5.12), we only need to prove

$$
\left\|\langle\nabla\rangle^{5} \nabla \frac{\partial_{x} \partial_{y}}{-\Delta} \partial_{x}\left(\partial_{y} P_{\leq s^{\delta}} \psi \partial_{x} \partial_{y} P_{\leq s^{\delta}} \psi\right)\right\|_{L_{x y}^{1}} \lesssim s^{-\frac{3}{2}-\varepsilon} Q\left(\|(\vec{u}, \psi)\|_{Y}\right) .
$$

Again,

$$
\begin{aligned}
& \left\|\langle\nabla\rangle^{5} \nabla \frac{\partial_{x} \partial_{y}}{-\Delta} \partial_{x}\left(\partial_{y} P_{\leq s^{\delta}} \psi \partial_{x} \partial_{y} P_{\leq s^{\delta}} \psi\right)\right\|_{L_{x y}^{1}} \\
& \lesssim\left\|\langle\nabla\rangle^{5+2 \epsilon}|\nabla|^{1-\epsilon} \frac{\partial_{x} \partial_{y}}{-\Delta} \partial_{x}\left(\partial_{y} P_{\leq s^{\delta}} \psi \partial_{x} \partial_{y} P_{\leq s^{\delta}} \psi\right)\right\|_{L_{x y}^{1}} \\
& \lesssim\left\|\partial_{x}\left(\partial_{y} P_{\leq s^{\delta}} \psi \partial_{x} \partial_{y} P_{\leq s^{\delta}} \psi\right)\right\|_{L_{x y}^{1}}^{2 \epsilon}\left\|\partial_{x}^{2}\left(\partial_{y} P_{\leq s^{\delta}} \psi \partial_{x} \partial_{y} P_{\leq s^{\delta}} \psi\right)\right\|_{L_{x y}^{1}}^{1-2 \epsilon} \\
& +\left\|\langle\nabla\rangle^{6+2 \epsilon} \partial_{x}^{2}\left(\partial_{y} P_{\leq s^{\delta}} \psi \partial_{x} \partial_{y} P_{\leq s^{\delta}} \psi\right)\right\|_{L_{x y}^{1}} \\
& \lesssim\left(s^{2 \epsilon\left(2 \delta-\frac{3}{2}\right)+(1-2 \epsilon)\left(2 \delta-\frac{7}{4}\right)}+s^{9 \delta-\frac{7}{4}}\right)\|(\vec{u}, \psi)\|_{Y}^{2} \\
& \lesssim s^{-\frac{3}{2}-\varepsilon}\|(\vec{u}, \psi)\|_{Y}^{2},
\end{aligned}
$$

where $\delta>0$ and satisfies $9 \delta+\varepsilon \leq \frac{1}{4}$. This gives (5.12). This finishes the proof of Lemma 5.4 . 


\section{Estimates on $\left\|\langle\nabla\rangle \partial_{x x} \psi\right\|_{\infty},\left\|\partial_{t} \vec{u}\right\|_{\infty},\left\|\langle\nabla\rangle \partial_{x} \vec{u}\right\|_{\infty}$}

This section continues the proof for (1.17). For the sake of clarity, we divide this section into subsections with each one of them devoted to one term. The tool lemmas in Section 2 will be used extensively here.

6.1. Estimate on $\left\|\langle\nabla\rangle \partial_{x x} \psi\right\|_{\infty}$. Using the Duhamel formula, namely (2.4),

$$
\psi(t, x, y)=K_{0}\left(t, \partial_{x}\right) \psi_{0}+K_{1}\left(t, \partial_{x}\right)\left(\frac{1}{2} \psi_{0}+\psi_{1}\right)+\int_{1}^{t} K_{1}\left(t-s, \partial_{x}\right) F_{0}(s) d s .
$$

For notational convenience, we may sometimes write $K_{0}(t)$ for $K_{0}\left(t, \partial_{x}\right)$ and $K_{1}(t)$ for $K_{1}\left(t, \partial_{x}\right)$. Therefore,

$$
\begin{gathered}
\left\|\langle\nabla\rangle \partial_{x x} \psi\right\|_{\infty} \lesssim \\
\left\|\langle\nabla\rangle \partial_{x x} K_{0}(t) \psi_{0}\right\|_{\infty}+\left\|\langle\nabla\rangle \partial_{x x} K_{1}(t)\left(\frac{1}{2} \psi_{0}+\psi_{1}\right)\right\|_{\infty} \\
+\left\|\int_{1}^{t}\langle\nabla\rangle \partial_{x x} K_{1}(t-s) F_{0}(s) d s\right\|_{\infty} .
\end{gathered}
$$

By Corollary 2.5] and Lemma 2.2,

$$
\begin{aligned}
& \left\|\langle\nabla\rangle \partial_{x x} K_{0}(t) \psi_{0}\right\|_{\infty} \\
\lesssim & \left(\left\|\widehat{\partial_{x x} K_{0}}(t, \xi)\right\|_{L_{\xi}^{1}\left(|\xi| \leq \frac{1}{2}\right)}+\left\|\widehat{K_{0}}(t, \xi)\right\|_{L_{\xi}^{\infty}\left(|\xi| \geq \frac{1}{2}\right)}\right)\left\|\langle\nabla\rangle^{2+\varepsilon} \partial_{x x} \partial_{y} \psi_{0}\right\|_{L_{x y}^{1}} \\
\lesssim & \left(t^{-\frac{3}{2}}+e^{-t}\right)\left\|\langle\nabla\rangle^{5+\varepsilon} \psi_{0}\right\|_{L_{x y}^{1}} \lesssim t^{-\frac{3}{2}}\left\|\langle\nabla\rangle^{5+\varepsilon} \psi_{0}\right\|_{X_{0}} .
\end{aligned}
$$

Since the estimates for $K_{0}$ and $K_{1}$ are the same, we also have

$$
\left\|\langle\nabla\rangle \partial_{x x} K_{1}(t)\left(\frac{1}{2} \psi_{0}+\psi_{1}\right)\right\|_{\infty} \lesssim t^{-\frac{3}{2}}\left\|\langle\nabla\rangle^{5+\varepsilon}\left(\frac{1}{2} \psi_{0}+\psi_{1}\right)\right\|_{X_{0}} .
$$

Moreover,

$$
\begin{aligned}
& \left\|\int_{1}^{t}\langle\nabla\rangle \partial_{x x} K_{1}(t-s) F_{0}(s) d s\right\|_{\infty} \\
\lesssim & \int_{1}^{t}\left\|\partial_{x x} K_{1}(t-s)\langle\nabla\rangle F_{0}(s)\right\|_{\infty} d s \\
\lesssim & \int_{1}^{\frac{t}{2}}\left\|\partial_{x x} K_{1}(t-s)\langle\nabla\rangle F_{0}(s)\right\|_{\infty} d s+\int_{\frac{t}{2}}^{t}\left\|\partial_{x} K_{1}(t-s)\langle\nabla\rangle \partial_{x} F_{0}(s)\right\|_{\infty} d s .
\end{aligned}
$$

By Corollary 2.5, Lemma 2.2 and Lemma 5.4,

$$
\begin{aligned}
& \int_{1}^{\frac{t}{2}}\left\|\partial_{x x} K_{1}(t-s)\langle\nabla\rangle F_{0}(s)\right\|_{\infty} d s \\
\lesssim & \int_{1}^{\frac{t}{2}}\left(\left\|\widehat{\partial_{x x} K_{1}}(t-s, \xi)\right\|_{L_{\xi}^{1}\left(|\xi| \leq \frac{1}{2}\right)}+\left\|\widehat{K_{1}}(t-s, \xi)\right\|_{L_{\xi}^{\infty}\left(|\xi| \geq \frac{1}{2}\right)}\right)\left\|\nabla\langle\nabla\rangle^{4+\varepsilon} F_{0}(s)\right\|_{L_{x y}^{1}} d s \\
\lesssim & \int_{1}^{\frac{t}{2}}\langle t-s\rangle^{-\frac{3}{2}}\left\|\nabla\langle\nabla\rangle^{4+\varepsilon} F_{0}(s)\right\|_{L_{x y}^{1}} d s \\
\lesssim & \int_{1}^{\frac{t}{2}}\langle t-s\rangle^{-\frac{3}{2}} s^{-\frac{3}{2}+\varepsilon} d s \cdot Q\left(\|(\vec{u}, \psi)\|_{Y}\right)
\end{aligned}
$$




$$
\lesssim t^{-\frac{3}{2}} Q\left(\|(\vec{u}, \psi)\|_{Y}\right)
$$

and

$$
\begin{aligned}
& \int_{\frac{t}{2}}^{t}\left\|\partial_{x} K_{1}(t-s)\langle\nabla\rangle \partial_{x} F_{0}(s)\right\|_{\infty} d s \\
\lesssim & \int_{\frac{t}{2}}^{t}\left(\left\|\widehat{\partial_{x} K_{1}}(t-s, \xi)\right\|_{L_{\xi}^{1}\left(|\xi| \leq \frac{1}{2}\right)}+\left\|\widehat{K_{1}}(t-s, \xi)\right\|_{L_{\xi}^{\infty}\left(|\xi| \geq \frac{1}{2}\right)}\right)\left\|\langle\nabla\rangle^{3+\varepsilon} \nabla \partial_{x} F_{0}(s)\right\|_{L_{x y}^{1}} d s \\
\lesssim & \int_{\frac{t}{2}}^{t}\langle t-s\rangle^{-1} s^{-\frac{3}{2}-\varepsilon} d s Q\left(\|(\vec{u}, \psi)\|_{Y}\right) \\
\lesssim & t^{-\frac{3}{2}} Q\left(\|(\vec{u}, \psi)\|_{Y}\right) .
\end{aligned}
$$

Combining the estimates above, we obtain

$$
\sup _{t \geq 1}\left(t^{\frac{3}{2}}\left\|\langle\nabla\rangle \partial_{x x} \psi(t)\right\|_{\infty}\right) \lesssim\left\|\left(\vec{u}_{0}, \psi_{0}\right)\right\|_{X_{0}}+Q\left(\|(\vec{u}, \psi)\|_{Y}\right) .
$$

6.2. Estimate of $\left\|\langle\nabla\rangle \partial_{t} \vec{u}\right\|_{\infty}$. Using the Duhamel formula, we obtain

$$
\partial_{t} u(t, x)=\dot{K}_{0}(t) u_{0}+\dot{K}_{1}(t)\left(\frac{1}{2} u_{0}+u_{1}\right)+\int_{1}^{t} \dot{K}_{1}(t-s) F_{1}(s) d s,
$$

then we have

$$
\begin{gathered}
\left\|\partial_{t}\langle\nabla\rangle u(t)\right\|_{\infty} \leq\left\|\dot{K}_{0}(t)\langle\nabla\rangle u_{0}\right\|_{\infty}+\left\|\dot{K}_{1}(t)\langle\nabla\rangle\left(\frac{1}{2} u_{0}+u_{1}\right)\right\|_{\infty} \\
+\left\|\int_{1}^{t} \dot{K}_{1}(t-s)\langle\nabla\rangle F_{1}(s) d s\right\|_{\infty} .
\end{gathered}
$$

For the linear parts, by Lemmas 2.2, 2.3 and 2.4, we have

$$
\begin{aligned}
\left\|\dot{K}_{0}(t)\langle\nabla\rangle u_{0}\right\|_{L_{\xi}^{\infty}} \lesssim\left\|\widehat{\dot{K}}_{0}(t, \xi)\right\|_{L_{\xi}^{1}\left(|\xi| \leq \frac{1}{2}\right)}\left\|\partial_{y}\langle\nabla\rangle u_{0}\right\|_{L_{x y}^{1}} & \\
& +\left\|\langle\xi\rangle^{-1} \widehat{\dot{K}}_{0}(t, \xi)\right\|_{L_{\xi}^{\infty}\left(|\xi| \geq \frac{1}{2}\right)}\left\|\partial_{y}\langle\nabla\rangle^{3+\epsilon} u_{0}\right\|_{L_{x y}^{1}} \\
\lesssim & t^{-\frac{3}{2}}\left\|\langle\nabla\rangle^{4+\epsilon} u_{0}\right\|_{L_{x y}^{1}} .
\end{aligned}
$$

Also we have,

$$
\left\|\dot{K}_{1}(t)\langle\nabla\rangle\left(\frac{1}{2} u_{0}+u_{1}\right)\right\|_{L_{x y}^{\infty}} \lesssim t^{-\frac{3}{2}}\left\|\langle\nabla\rangle^{3+\epsilon}\left(\frac{1}{2} u_{0}+u_{1}\right)\right\|_{L_{x y}^{1}} .
$$

For the nonlinear part, by Lemma 5.2 we have

$$
\begin{aligned}
\| \int_{1}^{t} \dot{K}_{1}(t-s) & \langle\nabla\rangle F_{1}(s) d s \|_{\infty} \lesssim \int_{1}^{t}\left(\left\|\widehat{\dot{K}}_{1}(t-s, \xi)\right\|_{L_{\xi}^{1}\left(|\xi| \leq \frac{1}{2}\right)}\right. \\
& \left.+\left\|{\widehat{\dot{K}_{1}}}_{1}(t-s, \xi)\right\|_{L_{\xi}^{\infty}\left(|\xi| \geq \frac{1}{2}\right)}\right) \cdot\left\|\langle\nabla\rangle^{2+\varepsilon} \nabla F_{1}(s)\right\|_{L_{x y}^{1}} d s \\
& \lesssim \int_{1}^{t}\langle t-s\rangle^{-\frac{3}{2}} s^{-\frac{3}{2}-\varepsilon} d s \cdot Q\left(\|(\vec{u}, \psi)\|_{Y}\right) \\
\lesssim & t^{-\frac{3}{2}} Q\left(\|(\vec{u}, \psi)\|_{Y}\right) .
\end{aligned}
$$


Combining with the estimates above, we deduce that

$$
\sup _{t \geq 1}\left(t^{\frac{3}{2}}\left\|\langle\nabla\rangle \partial_{t} u(t)\right\|_{\infty}\right) \lesssim\left\|\left(\vec{u}_{0}, \psi_{0}\right)\right\|_{X_{0}}+Q\left(\|(\vec{u}, \psi)\|_{Y}\right) .
$$

Similarly, by the Duhamel formula, we have

$$
\partial_{t} v(t, x)=\dot{K}_{0}(t) v_{0}+\dot{K}_{1}(t)\left(\frac{1}{2} v_{0}+v_{1}\right)+\int_{1}^{t} \dot{K}_{1}(t-s) F_{2}(s) d s .
$$

By using Lemma 5.3 instead, it obeys a similar estimate as the one for $u$. Therefore,

$$
\sup _{t \geq 1}\left(t^{\frac{3}{2}}\left\|\langle\nabla\rangle \partial_{t} \vec{u}(t)\right\|_{\infty}\right) \lesssim\left\|\left(\vec{u}_{0}, \psi_{0}\right)\right\|_{X_{0}}+Q\left(\|(\vec{u}, \psi)\|_{Y}\right) .
$$

6.3. Estimate of $\left\|\partial_{x}\langle\nabla\rangle \vec{u}(t)\right\|_{\infty}$. The estimate for $\left\|\partial_{x}\langle\nabla\rangle u(t)\right\|_{\infty}$ is similar as that on $\left\|\partial_{t}\langle\nabla\rangle u(t)\right\|_{\infty}$

$$
\begin{aligned}
\left\|\partial_{x}\langle\nabla\rangle u(t)\right\|_{\infty} \lesssim & \left\|\partial_{x} K_{0}(t)\langle\nabla\rangle u_{0}\right\|_{\infty}+\left\|\partial_{x} K_{1}(t)\langle\nabla\rangle\left(\frac{1}{2} u_{0}+u_{1}\right)\right\|_{\infty} \\
& +\left\|\int_{1}^{t} \partial_{x} K_{1}(t-s)\langle\nabla\rangle F_{1}(s) d s\right\|_{\infty} \\
\lesssim & t^{-1}\left(\left\|\langle\nabla\rangle^{3+\varepsilon} u_{0}\right\|_{L_{x y}^{1}}+\left\|\langle\nabla\rangle^{3+\varepsilon} u_{1}\right\|_{L_{x y}^{1}}\right) \\
& +\int_{1}^{t}\langle t-s\rangle^{-\frac{3}{2}} s^{-\frac{3}{2}-\varepsilon} d s \cdot Q\left(\|(\vec{u}, \psi)\|_{Y}\right) \\
\lesssim & t^{-1}\left(\left\|\left(\vec{u}_{0}, \psi_{0}\right)\right\|_{X_{0}}+Q\left(\|(\vec{u}, \psi)\|_{Y}\right)\right) .
\end{aligned}
$$

$\left\|\partial_{x}\langle\nabla\rangle v(t)\right\|_{\infty}$ can be bounded similarly as the one for $\left\|\partial_{x}\langle\nabla\rangle u(t)\right\|_{\infty}$. We omit the details. Thus,

$$
\sup _{t \geq 1}\left(t\left\|\langle\nabla\rangle \partial_{x} \vec{u}(t)\right\|_{\infty}\right) \lesssim\left\|\left(\vec{u}_{0}, \psi_{0}\right)\right\|_{X_{0}}+Q\left(\|(\vec{u}, \psi)\|_{Y}\right)
$$

7. Estimates ON $\left\|\langle\nabla\rangle^{3} \psi\right\|_{2},\left\|\langle\nabla\rangle^{3} \partial_{x}^{2} \psi\right\|_{2},\left\|\partial_{x}^{3} \psi\right\|_{2},\left\|\langle\nabla\rangle \partial_{t} \vec{u}\right\|_{2}$ AND $\left\|\partial_{x} \partial_{t} v\right\|_{2}$

The estimates on $\left\|\langle\nabla\rangle^{3} \psi\right\|_{2},\left\|\langle\nabla\rangle^{2} \partial_{x}^{2} \psi\right\|_{2},\left\|\partial_{x}^{3} \psi\right\|_{2},\left\|\langle\nabla\rangle \partial_{t} \vec{u}\right\|_{2}$ and $\left\|\partial_{x} \partial_{t} v\right\|_{2}$ can be similarly obtained as in section 6 .

By Lemmas 2.2, 2.6 and 2.7.

$$
\begin{aligned}
\left\|\langle\nabla\rangle^{3} \psi\right\|_{2} \lesssim & \left\|K_{0}(t)\langle\nabla\rangle^{3} \psi_{0}\right\|_{2}+\left\|K_{1}(t)\langle\nabla\rangle^{3}\left(\frac{1}{2} \psi_{0}+\psi_{1}\right)\right\|_{2} \\
& +\left\|\int_{1}^{t} K_{1}(t-s)\langle\nabla\rangle^{3} F_{0}(s) d s\right\|_{L^{2}} \\
\lesssim & t^{-\frac{1}{4}}\left(\left\|\langle\nabla\rangle^{4+\varepsilon} u_{0}\right\|_{L_{x y}^{1}}+\left\|\langle\nabla\rangle^{4+\varepsilon} u_{1}\right\|_{L_{x y}^{1}}\right)+\int_{1}^{t}\left(\left\|\widehat{K_{1}}(t-s, \xi)\right\|_{L_{\xi}^{2}\left(|\xi| \leq \frac{1}{2}\right)}\right. \\
& \left.+\left\|\widehat{K_{1}}(t-s, \xi)\right\|_{L_{\xi}^{\infty}\left(|\xi| \geq \frac{1}{2}\right)}\right)\left\||\nabla|^{\frac{1}{2}-\varepsilon}\langle\nabla\rangle^{\frac{7}{2}+2 \varepsilon} F_{0}(s)\right\|_{L_{x y}^{1}} d s \\
& \lesssim t^{-\frac{1}{4}}\left\|\left(\vec{u}_{0}, \psi_{0}\right)\right\|_{X_{0}}+\int_{1}^{t}\langle t-s\rangle^{-\frac{1}{4}} s^{-1-\varepsilon} d s \cdot Q\left(\|(\vec{u}, \psi)\|_{Y}\right)
\end{aligned}
$$




$$
\lesssim t^{-\frac{1}{4}}\left(\left\|\left(\vec{u}_{0}, \psi_{0}\right)\right\|_{X_{0}}+Q\left(\|(\vec{u}, \psi)\|_{Y}\right)\right) .
$$

We define a Fourier multiplier operator $\left|\partial_{x}\right|^{1-2 \varepsilon}$ as

$$
\left|\partial_{x}\right|^{1-2 \varepsilon} f(x, y)=\int e^{i x \xi+i y \eta}|\xi|^{1-2 \varepsilon} \widehat{f}(\xi, \eta) d \xi d \eta .
$$

Then as before, by Lemmas 2.2, 2.6 and 2.7, we have

$$
\begin{aligned}
& \left\|\langle\nabla\rangle^{3} \partial_{x}^{2} \psi\right\|_{2} \lesssim\left\|\partial_{x}^{2} K_{0}(t)\langle\nabla\rangle^{3} \psi_{0}\right\|_{2}+\left\|\partial_{x}^{2} K_{1}(t)\langle\nabla\rangle^{3}\left(\frac{1}{2} \psi_{0}+\psi_{1}\right) \psi_{0}\right\|_{2} \\
& +\left\|\int_{1}^{t} \partial_{x}^{2} K_{1}(t-s)\langle\nabla\rangle^{2} F_{0}(s) d s\right\|_{L^{2}} \\
& \lesssim t^{-\frac{5}{4}}\left(\left\|\langle\nabla\rangle^{6+\varepsilon} u_{0}\right\|_{L_{x y}^{1}}+\left\|\langle\nabla\rangle^{6+\varepsilon} u_{1}\right\|_{L_{x y}^{1}}\right)+\int_{1}^{\frac{t}{2}}\left\|\partial_{x}^{2} K_{1}(t-s)\langle\nabla\rangle^{2} F_{0}(s)\right\|_{L^{2}} d s \\
& +\int_{\frac{t}{2}}^{t}\left\|\left|\partial_{x}\right|^{\frac{3}{2}-2 \varepsilon} K_{1}(t-s)\langle\nabla\rangle^{2}\left|\partial_{x}\right|^{\frac{1}{2}+2 \varepsilon} F_{0}(s)\right\|_{L^{2}} d s \\
& \lesssim t^{-\frac{5}{4}}\left\|\left(\vec{u}_{0}, \psi_{0}\right)\right\|_{X_{0}}+\int_{1}^{\frac{t}{2}}\left(\left\|\widehat{\partial_{x}^{2} K_{1}}(t-s, \xi)\right\|_{L_{\xi}^{2}\left(|\xi| \leq \frac{1}{2}\right)}\right. \\
& \left.+\left\|\widehat{K_{1}}(t-s, \xi)\right\|_{L_{\xi}^{\infty}\left(|\xi| \geq \frac{1}{2}\right)}\right)\left\||\nabla|^{\frac{1}{2}-\varepsilon}\langle\nabla\rangle^{\frac{11}{2}+2 \varepsilon} F_{0}(s)\right\|_{L_{x y}^{1}} d s \\
& +\int_{\frac{t}{2}}^{t}\left(\left\||\xi|^{\frac{3}{2}-2 \varepsilon} \widehat{K_{1}}(t-s, \xi)\right\|_{L_{\xi}^{2}\left(|\xi| \leq \frac{1}{2}\right)}\right. \\
& \left.+\left\|\widehat{K_{1}}(t-s, \xi)\right\|_{L_{\xi}^{\infty}\left(|\xi| \geq \frac{1}{2}\right)}\right)\left\|\langle\nabla\rangle^{5+2 \varepsilon} \nabla F_{0}(s)\right\|_{L_{x y}^{1}} d s \\
& \lesssim t^{-\frac{5}{4}}\left\|\left(\vec{u}_{0}, \psi_{0}\right)\right\|_{X_{0}}+\int_{1}^{\frac{t}{2}}\langle t-s\rangle^{-\frac{5}{4}} s^{-1-\varepsilon} d s \cdot Q\left(\|(\vec{u}, \psi)\|_{Y}\right) \\
& +\int_{\frac{t}{2}}^{t}\langle t-s\rangle^{-1+\varepsilon} s^{-\frac{3}{2}+\varepsilon} d s \cdot Q\left(\|(\vec{u}, \psi)\|_{Y}\right) \\
& \lesssim t^{-\frac{5}{4}}\left(\left\|\left(\vec{u}_{0}, \psi_{0}\right)\right\|_{X_{0}}+Q\left(\|(\vec{u}, \psi)\|_{Y}\right)\right) .
\end{aligned}
$$

Now we consider $\left\|\partial_{x}^{3} \psi\right\|_{2}$. By Lemmas 2.2, 2.6 and 2.7.

$$
\begin{aligned}
\left\|\partial_{x}^{3} \psi\right\|_{2} \lesssim & \left\|\partial_{x}^{3} K_{0}(t) \psi_{0}\right\|_{2}+\left\|\partial_{x}^{3} K_{1}(t)\left(\frac{1}{2} \psi_{0}+\psi_{1}\right)\right\|_{2} \\
& +\left\|\int_{1}^{t} \partial_{x}^{3} K_{1}(t-s) F_{0}(s) d s\right\|_{L^{2}} \\
\lesssim & t^{-\frac{7}{4}}\left(\left\|\langle\nabla\rangle^{4+\varepsilon} u_{0}\right\|_{L_{x y}^{1}}+\left\|\langle\nabla\rangle^{4+\varepsilon} u_{1}\right\|_{L_{x y}^{1}}\right)+\int_{1}^{\frac{t}{2}}\left\|\partial_{x}^{3} K_{1}(t-s) F_{0}(s)\right\|_{L^{2}} d s \\
& +\int_{\frac{t}{2}}^{t}\left\|\left|\partial_{x}\right|^{\frac{3}{2}-2 \varepsilon} K_{1}(t-s)\left|\partial_{x}\right|^{\frac{3}{2}+2 \varepsilon} F_{0}(s)\right\|_{L^{2}} d s .
\end{aligned}
$$

We proceed in the same as in the previous estimate,

$$
\left\|\partial_{x}^{3} \psi\right\|_{2} \lesssim t^{-\frac{7}{4}}\left\|\left(\vec{u}_{0}, \psi_{0}\right)\right\|_{X_{0}}+\int_{1}^{\frac{t}{2}}\langle t-s\rangle^{-\frac{7}{4}}\left\||\nabla|^{\frac{1}{2}-\varepsilon}\langle\nabla\rangle^{\frac{7}{2}+2 \varepsilon} F_{0}(s)\right\|_{L_{x y}^{1}} d s
$$




$$
\begin{aligned}
& \quad+\int_{\frac{t}{2}}^{t}\langle t-s\rangle^{-1+\varepsilon}\left\|\nabla \partial_{x}\langle\nabla\rangle^{2+2 \varepsilon} F_{0}\right\|_{L_{x y}^{1}} d s \\
& \lesssim t^{-\frac{7}{4}}\left\|\left(\vec{u}_{0}, \psi_{0}\right)\right\|_{X_{0}}+\int_{1}^{\frac{t}{2}}\langle t-s\rangle^{-\frac{7}{4}} s^{-1-\varepsilon} d s \cdot Q\left(\|(\vec{u}, \psi)\|_{Y}\right) \\
& \quad+\int_{\frac{t}{2}}^{t}\langle t-s\rangle^{-1+\varepsilon} s^{-\frac{3}{2}-\varepsilon} d s \cdot Q\left(\|(\vec{u}, \psi)\|_{Y}\right) \\
& \lesssim t^{-\frac{3}{2}}\left(\left\|\left(\vec{u}_{0}, \psi_{0}\right)\right\|_{X_{0}}+Q\left(\|(\vec{u}, \psi)\|_{Y}\right)\right) .
\end{aligned}
$$

We now bound $\left\|\langle\nabla\rangle \partial_{t} \vec{u}\right\|_{2}$. By the Duhamel formula,

$$
\begin{aligned}
\left\|\langle\nabla\rangle \partial_{t} u\right\|_{2} \lesssim & \left\|\dot{K}_{0}(t)\langle\nabla\rangle u_{0}\right\|_{2}+\left\|\dot{K}_{1}(t)\langle\nabla\rangle\left(\frac{1}{2} u_{0}+u_{1}\right)\right\|_{2} \\
& +\left\|\int_{1}^{t} \dot{K}_{1}(t-s)\langle\nabla\rangle F_{1}(s) d s\right\|_{2}
\end{aligned}
$$

By Lemma 2.2.

$$
\begin{aligned}
\| \dot{K}_{0}(t)\langle\nabla\rangle & u_{0}\left\|_{2}+\right\| \dot{K}_{1}(t)\langle\nabla\rangle\left(\frac{1}{2} u_{0}+u_{1}\right) \|_{2} \\
& \lesssim t^{-\frac{5}{4}}\left(\left\|\langle\nabla\rangle^{3+\varepsilon} u_{0}\right\|_{L_{x y}^{1}}+\left\|\langle\nabla\rangle^{2+\varepsilon} u_{1}\right\|_{L_{x y}^{1}}\right) .
\end{aligned}
$$

By Lemmas 2.6, 2.7 and 5.2,

$$
\begin{aligned}
& \left\|\int_{1}^{t} \dot{K}_{1}(t-s)\langle\nabla\rangle F_{1}(s) d s\right\|_{2} \\
\lesssim & \int_{1}^{t}\left(\left\|\widehat{\dot{K}}_{1}(t-s)\right\|_{L_{\xi}^{2}\left(|\xi| \leq \frac{1}{2}\right)}+\left\|\widehat{\dot{K}_{1}}(t-s)\right\|_{L_{\xi}^{\infty}\left(|\xi| \geq \frac{1}{2}\right)}\right)\left\||\nabla|^{\frac{1}{2}-\varepsilon}\langle\nabla\rangle^{\frac{3}{2}+\varepsilon} F_{1}(s)\right\|_{2} \\
\lesssim & \int_{1}^{t}\langle t-s\rangle^{-\frac{5}{4}} s^{-\frac{3}{2}-\varepsilon} d s Q\left(\|(\vec{u}, \psi)\|_{Y}\right) \\
\lesssim & t^{-\frac{5}{4}} Q\left(\|(\vec{u}, \psi)\|_{Y}\right) .
\end{aligned}
$$

Therefore,

$$
\left\|\langle\nabla\rangle \partial_{t} u\right\|_{2} \lesssim t^{-\frac{5}{4}}\left(\left\|\left(\vec{u}_{0}, \psi_{0}\right)\right\|_{X_{0}}+Q\left(\|(\vec{u}, \psi)\|_{Y}\right)\right)
$$

Similarly,

$$
\begin{aligned}
\left\|\langle\nabla\rangle \partial_{t} v\right\|_{2} \lesssim & \left\|\dot{K}_{0}(t)\langle\nabla\rangle v_{0}\right\|_{2}+\left\|\dot{K}_{1}(t)\langle\nabla\rangle\left(\frac{1}{2} v_{0}+v_{1}\right)\right\|_{2} \\
& +\left\|\int_{1}^{t} \dot{K}_{1}(t-s)\langle\nabla\rangle F_{2}(s) d s\right\|_{2} \\
\lesssim & t^{-\frac{5}{4}}\left(\left\|\left(\vec{u}_{0}, \psi_{0}\right)\right\|_{X_{0}}+Q\left(\|(\vec{u}, \psi)\|_{Y}\right)\right)
\end{aligned}
$$

Moreover,

$$
\begin{aligned}
\left\|\partial_{x} \partial_{t} v\right\|_{2} \lesssim & \left\|\dot{K}_{0}(t) \partial_{x} v_{0}\right\|_{2}+\left\|\dot{K}_{1}(t) \partial_{x}\left(\frac{1}{2} v_{0}+v_{1}\right)\right\|_{2} \\
& +\left\|\int_{1}^{t} \partial_{x} \dot{K}_{1}(t-s) F_{2}(s) d s\right\|_{2}
\end{aligned}
$$




$$
\begin{aligned}
& \lesssim t^{-\frac{3}{2}}\left(\left\|\langle\nabla\rangle^{3+\varepsilon} v_{0}\right\|_{L_{x y}^{1}}+\left\|\langle\nabla\rangle^{2+\varepsilon} v_{1}\right\|_{L_{x y}^{1}}\right)+\int_{1}^{t} t^{-\frac{3}{2}}\left\||\nabla|^{\frac{1}{2}-\varepsilon}\langle\nabla\rangle^{\frac{3}{2}+2 \varepsilon} F_{2}\right\|_{L_{x y}^{1}} d s \\
& \lesssim t^{-\frac{3}{2}}\left\|\left(\vec{u}_{0}, \psi_{0}\right)\right\|_{X_{0}}+\int_{1}^{t}\langle t-s\rangle^{-\frac{3}{2}} s^{-\frac{3}{2}-\varepsilon} d s \cdot Q\left(\|(\vec{u}, \psi)\|_{Y}\right) \\
& \lesssim t^{-\frac{3}{2}}\left(\left\|\left(\vec{u}_{0}, \psi_{0}\right)\right\|_{X_{0}}+Q\left(\|(\vec{u}, \psi)\|_{Y}\right)\right) .
\end{aligned}
$$

\section{Appendix A.}

This appendix serves four purposes. It gives an explicit representation of $\Pi_{1}$ and $\Pi_{2}$, which has been used in the estimates of $F_{0}, F_{1}$ and $F_{2}$. It also provides the proofs of Lemma 5.1 and Lemma 5.5. Finally, the properties of the pressure are also given here.

A.1. Another expression of $\Pi_{j}, j=1,2$. This subsection writes out each term of $\Pi_{1}$ and $\Pi_{2}$ explicitly. $\Pi_{1}$ and $\Pi_{2}$ are previously represented in vector form in (1.8) and (1.9), respectively. Our key point here is that each term is written in a way that it possesses as many directives in the $x$-direction as possible. As seen from Lemma 2.2 in Section 2 , the more $x$-derivatives a term has, the faster it decays in time. This point has played an important role in the estimates of $F_{0}, F_{1}$ and $F_{2}$ in Section 5 .

$$
\begin{aligned}
\Pi_{1}= & -u \partial_{x} u-v \partial_{y} u+\frac{\partial_{x y}}{\Delta}\left(u \partial_{x} v\right)-\frac{\partial_{x y}}{\Delta}\left(v \partial_{x} u\right) \\
& +\frac{\partial_{x x}}{\Delta}\left(u \partial_{x} u\right)+\frac{\partial_{x x}}{\Delta}\left(v \partial_{y} u\right)-\partial_{x} \psi \partial_{x x} \psi-\partial_{x} \psi \partial_{y y} \psi \\
& +\frac{\partial_{x y}}{\Delta}\left(\partial_{y} \psi \partial_{x x} \psi\right)+\frac{\partial_{y y}}{\Delta}\left(\partial_{y} \psi \partial_{x y} \psi\right)+\frac{\partial_{x x}}{\Delta}\left(\partial_{x} \psi \partial_{x x} \psi\right)+\frac{\partial_{x x}}{\Delta}\left(\partial_{x} \psi \partial_{y y} \psi\right),
\end{aligned}
$$

and

$$
\begin{aligned}
\Pi_{2}= & -u \partial_{x} v-v \partial_{y} v+\frac{\partial_{x y}}{\Delta}\left(u \partial_{x} u\right)+\frac{\partial_{x y}}{\Delta}\left(v \partial_{y} u\right) \\
& +\frac{\partial_{y y}}{\Delta}\left(u \partial_{x} v\right)+\frac{\partial_{y y}}{\Delta}\left(v \partial_{y} v\right)-\partial_{y y} \psi \partial_{y} \psi-\partial_{x x} \psi \partial_{y} \psi \\
& +\frac{\partial_{y y}}{\Delta}\left(\partial_{x x} \psi \partial_{y} \psi\right)+\frac{\partial_{y y}}{\Delta}\left(\partial_{y y} \psi \partial_{y} \psi\right)+\frac{\partial_{x y}}{\Delta}\left(\partial_{x x} \psi \partial_{x} \psi\right)+\frac{\partial_{x y}}{\Delta}\left(\partial_{y y} \psi \partial_{x} \psi\right) \\
= & -\frac{\partial_{x x}}{\Delta}\left(u \partial_{x} v\right)-\frac{\partial_{x x}}{\Delta}\left(v \partial_{y} v\right)+\frac{\partial_{x x y}}{\Delta}(u u)+\frac{\partial_{x y y}}{\Delta}(u v)-\frac{\partial_{x x}}{\Delta}\left(\partial_{x x} \psi \partial_{y} \psi\right) \\
& -\frac{\partial_{x x}}{\Delta}\left(\partial_{y y} \psi \partial_{y} \psi\right)+\frac{1}{2} \frac{\partial_{x x y}}{\Delta}\left(\partial_{x} \psi \partial_{x} \psi\right)+\frac{\partial_{x y y}}{\Delta}\left(\partial_{x} \psi \partial_{y} \psi\right)-\frac{\partial_{x y}}{\Delta}\left(\partial_{y} \psi \partial_{x y} \psi\right) \\
= & -2 \frac{\partial_{x x}}{\Delta}\left(u \partial_{x} v\right)+\partial_{x}(u v)+2 \frac{\partial_{x y}}{\Delta}\left(u \partial_{x} u\right) \\
& -2 \frac{\partial_{x y}}{\Delta}\left(\partial_{y} \psi \partial_{x y} \psi\right)+\frac{\partial_{x y}}{\Delta}\left(\partial_{x} \psi \partial_{x x} \psi\right)+\frac{\partial_{y y}}{\Delta}\left(\partial_{y} \psi \partial_{x x} \psi\right)+\frac{\partial_{y y}}{\Delta}\left(\partial_{x} \psi \partial_{x y} \psi\right)
\end{aligned}
$$


A.2. Proof of Lemma 5.1. It is easily followed by the Littlewood-Paley's decomposition. Indeed,

$$
\begin{aligned}
\|\mathcal{R} f\|_{L_{x y}^{1}} & \leq \sum_{M \leq 1}\left\|P_{M} \mathcal{R} f\right\|_{L_{x y}^{1}}+\sum_{M \geq 1}\left\|P_{M} \mathcal{R} f\right\|_{L_{x y}^{1}} \\
& \leq \sum_{M \leq 1}\left\|P_{M} f\right\|_{L_{x y}^{1}}+\sum_{M \geq 1}\left\|P_{M} f\right\|_{L_{x y}^{1}} \\
& \lesssim \sum_{M \leq 1} M^{\epsilon}\left\|P_{M}|\nabla|^{-\epsilon} f\right\|_{L_{x y}^{1}}+\sum_{M \geq 1} M^{-\epsilon}\left\|P_{M}\langle\nabla\rangle^{\epsilon} f\right\|_{L_{x y}^{1}} \\
& \lesssim\left\||\nabla|^{-\epsilon}\langle\nabla\rangle^{2 \epsilon} f\right\|_{L_{x y}^{1}} .
\end{aligned}
$$

This prove Lemma 5.1 .

A.3. Proof of Lemma 5.5. By the Littlewood-Paley decomposition,

$$
\begin{aligned}
\left\||\nabla|^{\alpha}\langle\nabla\rangle^{N_{0}} \frac{\partial_{x} \partial_{y}}{-\Delta} f\right\|_{L_{x y}^{1}} \lesssim & \sum_{M \leq 1} M^{\alpha-1}\left\|\partial_{x} f\right\|_{L_{x y}^{1}}+\sum_{M \geq 1} M^{N_{0}+\alpha-1}\left\|\partial_{x} f\right\|_{L_{x y}^{1}} \\
\lesssim & \sum_{M \leq 1} M^{\frac{\epsilon}{\alpha-\epsilon}} M^{-1}\left\|\nabla \partial_{x} f\right\|_{L_{x y}^{1}}^{\alpha-\epsilon}\left\|\partial_{x} f\right\|_{L_{x y}^{1}}^{1-\alpha+\epsilon} \\
& +\sum_{M \geq 1} M^{-\epsilon}\left\|\langle\nabla\rangle^{N_{0}-1+\alpha+\epsilon} \partial_{x} f\right\|_{L_{x y}^{1}} \\
\lesssim & \sum_{M \leq 1} M^{\frac{\epsilon}{\alpha-\epsilon}}\left\|\partial_{x} f\right\|_{L_{x y}^{1}}^{\alpha-\epsilon}\|f\|_{L_{x y}^{1}}^{1-\alpha+\epsilon}+\left\|\langle\nabla\rangle^{N_{0}-1+\alpha+\epsilon} \partial_{x} f\right\|_{L_{x y}^{1}} \\
\lesssim & \lesssim f\left\|_{L_{x y}^{1}}^{1-\alpha+\epsilon}\right\| \partial_{x} f\left\|_{L_{x y}^{1}}^{\alpha-\epsilon}+\right\|\langle\nabla\rangle^{N_{0}-1+\alpha+\epsilon} \partial_{x} f \|_{L_{x y}^{1} .}
\end{aligned}
$$

This proves Lemma 5.5 .

A.4. Properties of the pressure $P$. First of all, by applying $\nabla \cdot \vec{u}=0$, we have

$$
\begin{aligned}
P & =\frac{\nabla \cdot[\nabla \cdot(\nabla \phi \otimes \nabla \phi+\vec{u} \otimes \vec{u})]}{-\Delta} \\
& =-2 \partial_{y} \phi+\frac{\nabla \cdot[\nabla \cdot(\nabla \psi \otimes \nabla \psi+\vec{u} \otimes \vec{u})]}{-\Delta} .
\end{aligned}
$$

Therefore, for any $t \geq 1$, by Sobolev' inequality,

$$
\begin{aligned}
\|P(t)\|_{H^{N}} & \lesssim\left\|\partial_{y} \psi\right\|_{H^{N}}+\|\nabla \psi \otimes \nabla \psi\|_{H^{N}}+\|\vec{u} \otimes \vec{u}\|_{H^{N}} \\
& \lesssim\|\nabla \psi\|_{H^{N}}+\|\nabla \psi\|_{\infty}\|\nabla \psi\|_{H^{N}}+\|\vec{u}\|_{\infty}\|\vec{u}\|_{H^{N}} \\
& \lesssim t^{\varepsilon}\left(\|(\vec{u}, \psi)\|_{Y}+\|(\vec{u}, \psi)\|_{Y}^{2}\right) \\
& \lesssim \varepsilon_{0} t^{\varepsilon} .
\end{aligned}
$$

This implies that $P \in C\left([1, \infty) ; H^{N}\left(\mathbb{R}^{2}\right)\right)$. Furthermore,

$$
\begin{aligned}
\|P(t)\|_{\infty} & \lesssim\left\|\partial_{y} \psi\right\|_{\infty}+\left\|\langle\nabla\rangle^{2}(\nabla \psi \otimes \nabla \psi)\right\|_{2}+\left\|\langle\nabla\rangle^{2}(\vec{u} \otimes \vec{u})\right\|_{2} \\
& \lesssim\|\nabla \psi\|_{\infty}+\left\|\langle\nabla\rangle^{3} \psi\right\|_{2}\|\nabla \psi\|_{\infty}+\left\|\langle\nabla\rangle^{2} \vec{u}\right\|_{2}\|\vec{u}\|_{\infty} \\
& \lesssim t^{-\frac{1}{2}}\left(\|(\vec{u}, \psi)\|_{Y}+\|(\vec{u}, \psi)\|_{Y}^{2}\right) \\
& \lesssim \varepsilon_{0} t^{-\frac{1}{2}}
\end{aligned}
$$




\section{ACKNOWLEDGEMENTS}

J. Wu was partially supported by NSF grant DMS1209153. J. Wu thanks Professor Chongsheng Cao for discussions and the School of Mathematical Sciences, Beijing Normal University for its hospitality. Y. Wu was partially supported by the NSFC (No.11101042). Xu was partially supported by NSFC (No.11371059), BNSF (No.2112023). $\mathrm{Y}$. Wu and $\mathrm{Xu}$ were both partially supported by the Fundamental Research Funds for the Central Universities of China.

\section{REFERENCES}

[1] D. Biskamp, Nonlinear Magnetohydrodynamics, Cambridge University Press, Cambridge, 1993.

[2] I. Bejenaru and T. Tao, Sharp well-posedness and ill-posedness results for a quadratic non-linear Schrödinger equation, J. Funct. Anal. 233(2006), 228-259.

[3] C. Cao, D. Regmi and J. Wu, The 2D MHD equations with horizontal dissipation and horizontal magnetic diffusion, J. Differential Equations 254 (2013), 2661-2681.

[4] C. Cao, D. Regmi, J. Wu and X. Zheng, Global regularity for the 2D MHD equations with horizontal dissipation and horizontal magnetic diffusion, preprint.

[5] C. Cao and J. Wu, Global regularity for the 2D MHD equations with mixed partial dissipation and magnetic diffusion, Adv. Math. 226 (2011), 1803-1822.

[6] C. Cao, J. Wu and B. Yuan, The 2D incompressible magnetohydrodynamics equations with only magnetic diffusion, arXiv:1306.3629v1 [math.AP] 16 Jun 2013.

[7] Q. Chen, C. Miao and Z. Zhang, The Beale-Kato-Majda criterion for the 3D magnetohydrodynamics equations, Comm. Math. Phys. 275 (2007), 861-872.

[8] Q. Chen, C. Miao and Z. Zhang, On the well-posedness of the ideal MHD equations in the TriebelLizorkin spaces, Arch. Ration. Mech. Anal. 195 (2010), 561-578.

[9] P.A. Davidson, An Introduction to Magnetohydrodynamics, Cambridge University Press, Cambridge, 2001.

[10] G. Duvaut and J.-L. Lions, Inéquations en thermoélasticité et magnétohydrodynamique, Arch. Ration. Mech. Anal. 46 (1972), 241-279.

[11] J. Fan, G. Nakamura and Y. Zhou, Global Cauchy problem of 2D generalized MHD equations, preprint.

[12] C. He and Z. Xin, Partial regularity of suitable weak solutions to the incompressible magnetohydrodynamic equations, J. Funct. Anal. 227 (2005), 113-152.

[13] C. He and Z. Xin, On the regularity of weak solutions to the magnetohydrodynamic equations, $J$. Differential Equations 213 (2005), 235-254.

[14] Q. Jiu and D. Niu, Mathematical results related to a two-dimensional magneto-hydrodynamic equations, Acta Math. Sci. Ser. B Engl. Ed. 26 (2006), 744-756.

[15] Q. Jiu and J. Zhao, A remark on global regularity of 2D generalized magnetohydrodynamic equations, arXiv:1306.2823v1 [math.AP] 13 Jun 2013.

[16] Q. Jiu and J. Zhao, Global regularity of 2D generalized MHD equations with magnetic diffusion, arXiv:1309.5819 [math.AP] 23 Sep 2013.

[17] Z. Lei, On Axially Symmetric incompressible magnetohydrodynamics in three dimensions, arXiv:1212.5968 [math.AP] 24 Dec 2012.

[18] Z. Lei and Y. Zhou, BKM's criterion and global weak solutions for magnetohydrodynamics with zero viscosity, Discrete Contin. Dyn. Syst. 25 (2009), 575-583.

[19] D. Li and Y. Wu, The Cauchy problem for the two dimensional Euler-Poisson system, J. Eur. Math. Soc., to appear.

[20] F. Lin, L. Xu, and P. Zhang, Global small solutions to 2-D incompressible MHD system, arXiv:1302.5877v2 [math.AP] 4 Jun 2013.

[21] F. Lin and P. Zhang, Global small solutions to MHD type system (I): 3-D case, Comm. Pure. Appl. Math., to appear. 
[22] E. Priest and T. Forbes, Magnetic reconnection, MHD theory and Applications, Cambridge University Press, Cambridge, 2000.

[23] M. Sermange and R. Temam, Some mathematical questions related to the MHD equations, Comm. Pure Appl. Math. 36 (1983), 635-664.

[24] C. Trann, X. Yu and Z. Zhai, On global regularity of 2D generalized magnetohydrodynamic equations, J. Differential Equations 254 (2013), 4194-4216.

[25] W. Wang and Z. Zhang, Limiting case for the regularity criterion to the 3-D magnetohydrodynamics equations, J. Differential Equations 252 (2012), 5751-5762.

[26] J. Wu, Generalized MHD equations, J. Differential Equations 195 (2003), 284-312.

[27] J. Wu, Regularity criteria for the generalized MHD equations, Comm. Partial Differential Equations 33 (2008), 285-306.

[28] J. Wu, Global regularity for a class of generalized magnetohydrodynamic equations, J. Math. Fluid Mech. 13 (2011), 295-305.

[29] L. Xu and P. Zhang, Global small solutions to three-dimensional incompressible MHD system, preprint.

[30] B. Yuan and L. Bai, Remarks on global regularity of 2D generalized MHD equations, arXiv:1306.2190v1 [math.AP] 11 Jun 2013.

[31] K. Yamazaki, Remarks on the global regularity of two-dimensional magnetohydrodynamics system with zero dissipation, arXiv:1306.2762 v1 [math.AP] 13 Jun 2013.

[32] K. Yamazaki, On the global regularity of two-dimensional generalized magnetohydrodynamics system arXiv:1306.2842v1 [math.AP] 13 Jun 2013.

[33] Y. Zhou and J. Fan, A regularity criterion for the 2D MHD system with zero magnetic diffusivity, J. Math. Anal. Appl. 378 (2011), 169-172.

1 Department of Mathematics, Oklahoma State University, 401 Mathematical Sciences, Stillwater, OK 74078, USA; and Department of Mathematics, College of NatuRal Science, Chung-Ang University, Seoul 156-756, Korea

E-mail address: jiahong@math.okstate.edu

${ }^{2}$ School of Mathematical Sciences, Beijing Normal University and Laboratory of Mathematics and Complex Systems, Ministry of Education, Beijing 100875, P.R. China

E-mail address: yifei@bnu.edu.cn; xjxu@bnu.edu.cn 